\title{
Ultra fast tissue staining with chemical tags
}

May 19, 2014

\author{
Johannes Kohl ${ }^{1}$, Julian $\mathrm{Ng}^{1}$, Sebastian Cachero ${ }^{1}$, Michael-John Dolan ${ }^{1}$, Ben Sutcliffe ${ }^{1}$, Daniel Krueger ${ }^{1}$, \\ Shahar Frechter ${ }^{1}$, Gregory S.X.E. Jefferis ${ }^{1}$ \\ ${ }^{1}$ Division of Neurobiology, MRC Laboratory of Molecular Biology, Cambridge, CB2 0QH, UK.
}

\begin{abstract}
Genetically encoded fluorescent proteins and immunostainings are widely used to detect cellular or subcellular structures in thick biological samples. However, each approach suffers from limitations, including low signal and limited spectral flexibility or slow speed, poor penetration and high background, respectively. Here we overcome these limitations by using transgenically expressed chemical tags for rapid, even and lowbackground labeling of thick biological tissues. We construct a platform of widely applicable transgenic Drosophila reporter lines, demonstrating that chemical labeling can accelerate staining of whole-mount fly brains by a factor of $100 \times$. Together, this tag-based approach drastically improves the speed and specificity of labeling genetically marked cells in intact and/or thick biological samples.
\end{abstract}




\section{INTRODUCTION}

The revolution in live imaging due to genetically encoded fluorescent proteins (FPs) is widely appreciated ${ }^{1,2}$, but FPs have also had a major impact on studies of fixed whole mount specimens or thick sections. Here immediate visualization, low background and spatially even signal are major advantages. However, FP signals are typically weaker, easily quenched by fixation and suffer from limited spectral flexibility. Therefore, antibody detection of marker proteins remains essential in many experimental situations. This tradeoff between uneven, slow staining and comparably high background levels due to antibody approaches, and the convenient but weaker, more labile and spectrally less diverse signals obtainable with FPs is central to many recent technical developments (e.g. array tomography ${ }^{3}, \mathrm{CLARITY}^{4}, \mathrm{Scale}^{5}$, SeeDB ${ }^{6}$ and $\mathrm{CUBIC}^{7}$ ), but remains fundamentally unresolved.

This tradeoff is also a major practical issue in neural circuit tracing in Drosophila ${ }^{8-13}$. We therefore sought staining methods that combine the positive aspects of both FP and antibody-based staining, notably fast, even, strong signal vs. low-background labeling. We have developed an approach based on four commercially available, orthogonal labeling chemistries (SNAP-, CLIP-, Halo- and TMP-tag) characterized by the covalent binding of a large range of (fluorescent) substrates to engineered enzyme tags. In order to use these chemistries for effective tissue labeling, we have generated the first stable transgenic reporter animals bearing these tags. We validate their use and expression in Drosophila, presenting 12 widely applicable fly strains for labeling of cells and sub-cellular structures. Combining these tags with the first knock-in construct, we demonstrate that our approach can speed up the most widely-used antibody staining procedure in Drosophila neurobiology ${ }^{12,14}$ from 1 week to 1 hour, a factor of $>100$, while giving more even staining and reduced background signals.

In conclusion, the chemical labeling reagents that we have developed and validated solve a basic but pervasive problem in tissue labeling and have immediate applications across model organisms and experimental disciplines.

\section{RESULTS}

\section{Expression of chemical tags in the Drosophila brain}

We sought to develop a labeling system that overcomes the limitations of antibody-based immunostainings, i.e. speed (poor penetration of thick tissue samples), specificity (background staining due to off-target binding) and complexity (number of user interactions, i.e. manual steps, in staining protocols).

Existing chemical tagging systems were compared and four were chosen that (1) do not require co-factors, (2) result in formation of a covalent bond and (3) for which a commercially available, spectrally diverse, range of fluorescent substrates is available. SNAP- and CLIP-tag ${ }^{15-18}$ (NEB), Halo-tag ${ }^{19,20}$ (Promega) and TMP$\operatorname{tag}^{21-23}$ (Active Motif) fulfilled these requirements. All four rely on the rapid $\left(10^{2}\right.$ to $\left.10^{6} \mathrm{M}^{-1} \mathrm{~s}^{-1}\right)$ formation of a covalent bond between an engineered, bio-orthogonal enzyme (tag) and a small reactive group which is fused to a reporter (substrate) ${ }^{24}$. SNAP- and CLIP-tag are modified, 181 amino acid (aa) $\mathrm{O}^{6}$-alkylguanine-DNA alkyltransferases that bind benzylguanine (BG) or benzylcytosine (BC) derivatives, respectively. Halo-tag (295 aa) is an engineered bacterial haloalkane dehalogenase that binds chloroalkane groups and TMP-tag (159 aa) is an $E$. coli dihydrofolate reductase (eDHFR) which has been engineered to covalently bind trimethoprim (TMP) derivatives (see Fig. 1a).

We made fusion constructs of these tags with membrane- or cytosolic proteins and cloned them downstream of a Gal4 UAS sequence ${ }^{25}$. The constructs were then injected into Drosophila melanogaster, generating a total of 12 transgenic lines (see Table S1).

We tested expression of these new fusion constructs using three different Gal4 driver lines: $\mathrm{fru}^{\mathrm{Gal} 4}$, which drives expression in about 2,000 fruitless-positive neurons ${ }^{26}$, GH146-Gal4, which expresses in most olfactory projection neurons ${ }^{27}$ and Mz19-Gal4 which is expressed in three classes of projection neurons, DA1, VA1d and $\mathrm{DC}^{28}$. As a reference, each driver line was crossed to flies expressing membrane-targeted GFP (myrGFP). When brains from the progeny of these driver-tag crosses were incubated with fluorescent SNAP-, CLIP-, Halo- 
or TMP substrates (see Table S2), strong, specific labeling was observed (Fig. 1b and Fig. S1). We tested all tag-substrate combinations for potential cross-reactivity and found no signal from non-cognate tag-substrate pairs, with the exception of weak binding of CLIP substrates to SNAP-tag (Fig. S2). This is expected since it has previously been shown that there is a $~ 100$-fold preference of BC substrates for CLIP (an engineered version of SNAP) over SNAP, as opposed to a > 1,000-fold preference of BG groups for SNAP over CLIP) ${ }^{16}$. Because of this low cross-reactivity and the availability of spectrally diverse substrates (Fig. 1c), this labeling approach potentially allows the simultaneous visualization of up to four orthogonal channels.

Having demonstrated efficient plasma membrane labeling, we then constructed synaptically localized tags, comparing them with established synaptic markers in Drosophila. Simultaneous immunostaining against the synaptotagmin-HA presynaptic marker (SytHA ${ }^{8,29,30}$ ) and chemical labeling with a SytCLIP construct revealed that chemical labeling consistently achieved higher signal-to-noise ratios with identical marker localization (Fig. 1d, top). This was even more pronounced when the somatodendritic marker TLN-mCherry (DenMark ${ }^{31}$ ) was compared to a TLN-SNAP construct (Fig. 1d, bottom). We used these synaptic markers to simultaneously label pre- and postsynaptic compartments of DA1 projection neurons with high signal-to-noise ratio (Fig. 1e). This highlights that by being bio-orthogonal, the chemical labeling approach can overcome one of the most common shortcomings of immunohistochemistry: high background staining due to poor epitope-specificity and/or cross-reactivity.

So far we have demonstrated (a) that chemical tags can be efficiently and specifically expressed in the Drosophila brain, (b) that these tags can be simultaneously used thanks to their orthogonality and the spectral range of their fluorescent substrates and (c) that the signal-to-noise ratio of this chemical labeling approach is superior to that commonly achieved with antibody staining.

\section{Ultrafast labeling of thick tissue samples}

Low fluorescence after fixation is a widely appreciated limitation of genetically encoded FPs ${ }^{7,32,33}$. Indeed, we observed that most fluorescence was quenched in GH146-Gal4 > myrGFP brains after standard fixation with PFA (Fig. S5f). Therefore, immunostainings are usually required to obtain sufficient signal from fixed samples. Antibody diffusion into tissue is another rate-limiting step for immunostainings of thick specimens. For example, homogeneous immunostaining of Drosophila brains (approximately $500 \times 250 \times 200 \mu \mathrm{m}$ in $x, y$ and $z$ ) for the nc82/Bruchpilot synaptic protein ${ }^{34}$ (the standard counterstain for brain structure in Drosophila neuroanatomical studies, see below) takes seven days (Fig. 2a) and requires $>15$ user interactions. This comprises a blocking step and prolonged incubations with primary and secondary antibodies ${ }^{9,12}$. These long staining times are due to the relatively large size of antibodies $(\sim 150 \mathrm{kDa})$, which limits their diffusion into thick tissue samples. We reasoned that the much smaller size $(\sim 1 \mathrm{kDa})$ of fluorescent chemical labeling substrates would considerably speed up the staining process.

We directly compared the labeling speed of antibodies and chemical substrates in fly brains expressing either membrane-targeted GFP or membrane-targeted SNAP-tag in olfactory projection neurons (PNs). Brains were dissected, fixed and briefly permeabilized, then incubated with GFP antibody or fluorescent SNAP substrate for $15 \mathrm{~min}, 30 \mathrm{~min}, 1 \mathrm{~h}, 4 \mathrm{~h}$ or $12 \mathrm{~h}$ (see Methods). After a washing step, fluorophore-conjugated antibody was added to brains expressing GFP for $2 \mathrm{~d}$. Brains expressing SNAP-tag were briefly washed and directly mounted. In a separate time series experiment, the incubation times in primary and secondary antibodies were identical (Fig. S5a). Whereas homogeneous staining of PNs with anti-GFP antibody required $>4 \mathrm{~h}$ primary antibody followed by $2 \mathrm{~d}$ of secondary antibody (Fig. 2b, top), strong and uniform chemical labeling was visible after only $15 \mathrm{~min}$ (Fig. 2b, middle). Such rapid labeling was also observed when using membrane-targeted Halotag (Fig. 2b, bottom). Quantification of staining intensity (see Methods) revealed that while antibody staining increased over the course of $12 \mathrm{~h}$, chemical staining levels reached near-maximum levels after $15 \mathrm{~min}$ (Fig. 2d). These results show that the small substrate size indeed leads to drastically reduced staining times with chemical labeling. Because no blocking step and far fewer washing steps were required for chemical labeling, the protocol comprised half as many user interactions (Fig. 2a).

We next assessed labeling uniformity and speed for the nc82/Bruchpilot antigen ${ }^{34}$; we compared nc82 
antibody labeling of wild-type (Canton-S) brains with labeling of a SNAP-tag knock-in construct inserted into the brp locus (brp-SNAP, see Fig. S4). Strikingly, while nc82 immunostaining for $12 \mathrm{~h}$ (primary) followed by 2 days (secondary) still resulted in substantial signal drop-off in the center of the brain (Fig. 2c, top), chemical labeling produced uniform staining within $15 \mathrm{~min}$ (Fig. 2c, bottom). Indeed we found that staining times of only 1 min were sufficient (Fig. S5d). Longer incubations did not appreciably increase neuropil labeling but instead resulted in higher background (visible in Fig. 2c, bottom right panel); this non-specific labeling could be removed by extended washes (see Fig. S6). This indicates that most tag molecules are occupied by substrate after minutes of incubation. We directly tested this by sequential labeling of the same sample with two different substrates (Fig. S6).

Using immunohistochemistry, we found that labeling the center of the brain $(\sim 100 \mu \mathrm{m}$ from surface $)$ required at least $12 \mathrm{~h}$ of primary followed by $2 \mathrm{~d}$ of secondary antibody incubation (Fig. 2e). In contrast, fluorescent substrates uniformly labeled the sample within less than $15 \mathrm{~min}$ (Fig. 2e). Therefore, the chemical staining approach enables rapid and homogeneous staining of thick tissue samples.

Finally, we tested the effect of fixation on the labeling reaction, incubating samples for 20, 40 or $60 \mathrm{~min}$ in $4 \%$ paraformaldehyde before adding substrates. We found that while SNAP- and CLIP-tag were largely insensitive to prolonged fixation, Halo-tag labeling decreased and TMP-tag labeling increased with longer fixation (Fig. S3). We did not observe fixation-dependent changes in background labeling (Fig. S3 and data not shown).

These results show that chemical labeling of thick tissue samples is at least two orders of magnitude faster than immunostainings and requires half as many user interactions, while at the same time resulting in better tissue labeling (Fig. 2a and Table 1).

\section{Applications in Drosophila Neurobiology}

The Drosophila reagents that we have described here have immediate and widespread utility in cell, developmental and neurobiology. In order to demonstrate this more clearly we optimized Drosophila transgenics for applications that are at the heart of large scale studies of neural circuits in the fly brain. The nc 82 marker is the most widely used counterstain for the Drosophila brain (currently 753 publications listed in Google Scholar http:

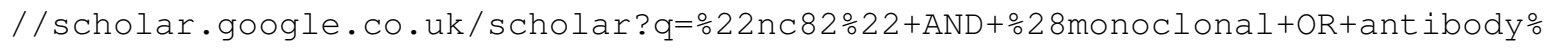
$\left.29 \& b \operatorname{nG}=\& h l=e n \& a s \_s d t=0 \div 2 \mathrm{C} 5\right)$. It has been used to construct standard brains for 3D atlases by image registration ${ }^{8,9,12,35}$ and is the basis of a $3 \mathrm{D}$ model encapsulating the recently standardized nomenclature for Drosophila brain regions ${ }^{36,37}$ (see also http://www. virtualflybrain.org). Homogeneous staining of neuropil structures throughout the brain is absolutely critical for successful registration ${ }^{9,38}$.

We first verified that the new brp-SNAP knock-in (Fig. 3a) closely recapitulates the neuropil staining obtained with nc82 antibody (Fig. 3b). We then confirmed that chemically labeled brains can easily be registered onto an existing nc82-immunostained template brain (IS2 $\left.{ }^{9}\right)$ with $100 \%$ success $(n=84$ specimens). Therefore, using the chemical brp-SNAP counterstain, rapidly labeled brains from different experimental sources can be comfortably registered into the same reference space, enabling direct comparison of labeled structures. In order to demonstrate this we generated a fly line bearing both a UAS-myrHalo reporter gene and brp-SNAP, crossed it to the sparse Mz19-Gal4 line and registered the brains of progeny after 30 min of labeling (Fig. 3c). We were subsequently able to resolve fine details of individual neurites and presynaptic boutons in the labeled $\mathrm{Mz} 19$ Gal4 expression pattern (Fig. 3c). Even when crossed to comparatively weak driver lines our chemical labeling reporter lines gave strong enough signal to visualize single neurons (Fig. 3c). Another powerful technique for sparse labeling of neuronal subsets within a wider expression pattern is the use of photoactivatable GFP (PAGFP); here spatially targeted laser stimulation can identify neurons with processes in a specific region of the brain ${ }^{39,40}$. However, although these methods effectively reveal neuronal morphology, they cannot easily be used with image registration approaches since the PA-GFP signal does not survive standard neuropil immunostaining protocols. We demonstrate that $b r p-S N A P$ labeling can be used to substitute for nc82 labeling in this context as well, allowing co-registration of PA-GFP specimens (Fig. 3c).

All of these newly generated data can be integrated with existing resources defined by registration against nc82 template brains, such as stochastically labeled single neurons ${ }^{38}$, lineage clones ${ }^{9,41,42}$, large-scale expres- 
sion screens ${ }^{12}$, single recorded neurons ${ }^{13}$ or the standard neuropil regions defined by the Insect Brain Name working group ${ }^{37}$ (Fig. 3c). We demonstrated this important point by co-registration of chemically labeled Mz19-Gal4 DA1 projection neurons with a dye-filled third-order olfactory neuron ${ }^{13}$ (Fig. 3d).

\section{DISCUSSION}

This work aims to address a fundamental need for visualizing genetically marked cellular structures in thick or intact tissue samples with high signal-to-noise ratio. It combines advantages of immunostainings (spectral flexibility, high signal after fixation) and genetically encoded fluorophores (low background) while drastically reducing staining time. The approach presented here is practical: it utilizes extensively tested and commercially available reagents in a novel context. Thus, little or no optimization will be required by researchers wishing to introduce this method. Because the building blocks for coupling reporter molecules to reactive groups are also available, customized substrates can be made by individual groups. Use of suitable probes should allow to combine chemical labeling with electron or super-resolution microscopy in the future. One advantage of chemical labeling in this context is its inherent linearity (i.e. one substrate molecule binds to one tag molecule), in contrast to often highly non-linear immunostainings. Chemical labeling of appropriate cellular targets is therefore especially suited for quantitative studies.

While our approach requires the use of transgenic animals and therefore can not replace the use of antibodies against specific cellular proteins, recent advances in molecular biology ${ }^{43-46}$ have greatly facilitated the generation of even complex DNA constructs. Furthermore, the CRISPR method now enables the very rapid generation of transgenic animals at defined loci, either for introducing reporter genes or tagging endogenous proteins ${ }^{47-53}$.

Our experiments show that SNAP-, Halo- and CLIP-tags are suitable for single-cell labeling. In contrast, using TMP-tag for this purpose will require further optimization. Other, orthogonal, labeling chemistries such as ACP-tag ${ }^{54}$ could be explored in the future to allow simultaneous visualization of $>4$ channels. The quantitative nature of chemical labeling also makes it particularly suitable for multiplexing strategies such as Brainbow ${ }^{55}$. Two Brainbow approaches are currently available in flies ${ }^{32,56}$. In our hands antibody staining against dBrainbow ${ }^{56}$ proteins is too variable across the brain to enable segmentation of neurons by color; conversely, Flybow ${ }^{32}$ fluorescent proteins have insufficient signal-to-noise ratio for mapping individual neurons in the central brain. The data in this paper suggest that chemical labeling would overcome these difficulties. We are currently working on such approaches for rapid, multicolor labeling of neuronal tissue in flies and mice.

We have generated transgenic fly lines suitable for a wide range of applications in cell and developmental biology (see Table S1). In the field of neurobiology, using membrane-targeted reporter lines in combination with $b r p-S N A P$ will considerably speed up anatomical studies where high resolution confocal imaging requires fixed and stained specimens. Furthermore, although intensity-based image registration has been used to great effect in large-scale anatomical studies ${ }^{8,9,11,12}$, it has not yet been widely adopted - one major factor being the difficulty of obtaining successful neuropil staining using nc 82 antibody.

We have noticed that the limiting factor for spatially even immunostaining of thick samples is diffusion of secondary antibody into the tissue: while antibody-penetration into fly brains is incomplete after $12 \mathrm{~h}$ incubation with each primary and secondary antibody (Fig. S5b), prolonging secondary incubation to two days results in more homogeneous labeling (Fig. 2c, top). Indeed, others have recommended extending secondary antibody incubations to four days ${ }^{12}$. Arguably, incubation times are less important in a high-throughput context. However, our approach requires half as many manual handling steps, resulting in proportional labor and cost savings. In any case, reducing a standard staining time from one week to one hour results in a shortening of the experimental cycle that has major benefits: conditions can be rapidly optimized and experimental decisions can be made faster. Furthermore, we find that inexperienced users can obtain even and robust labeling with this method whereas e.g. nc82 immunostaining is subject to batch-to-batch variation, even in experienced hands.

Rapid staining may be of even greater significance in larger specimens. For example several recent methods can render tissue sufficiently transparent to enable whole-mount optical imaging of mouse brains ${ }^{4-7}$. How- 
ever, high-resolution, multichannel imaging of fluorescent proteins has major limitations in sensitivity. The recently developed CLARITY technique additionally promises to enable antibody staining of intact brain tissue $^{4}$. Nonetheless, the reported staining time of four weeks for an adult mouse brain $(\sim 5 \mathrm{~mm}$ across, i.e. 2.5 $\mathrm{mm}$ from each surface) will likely be prohibitive for many experiments and there are still signs of significant spatial inhomogeneity. Extrapolating from our results in $\sim 200 \mu \mathrm{m}$ fly brains, chemical labeling could reduce this step to $<3$ hours, a more than 200 -fold improvement, while enabling more spatially even labeling. We are presently investigating the use of chemical labeling in mice. 


\section{References}

[1] R Y Tsien. The green fluorescent protein. Anпи Rev Biochem, 67:509-44, 1998.

[2] M Chalfie. Green fluorescent protein. Photochem Photobiol, 62(4):651-6, Oct 1995.

[3] Kristina D Micheva and Stephen J Smith. Array tomography: a new tool for imaging the molecular architecture and ultrastructure of neural circuits. Neuron, 55(1):25-36, Jul 2007.

[4] Kwanghun Chung, Jenelle Wallace, Sung-Yon Kim, Sandhiya Kalyanasundaram, Aaron S Andalman, Thomas J Davidson, Julie J Mirzabekov, Kelly A Zalocusky, Joanna Mattis, Aleksandra K Denisin, Sally Pak, Hannah Bernstein, Charu Ramakrishnan, Logan Grosenick, Viviana Gradinaru, and Karl Deisseroth. Structural and molecular interrogation of intact biological systems. Nature, 497(7449):332-7, May 2013.

[5] Hiroshi Hama, Hiroshi Kurokawa, Hiroyuki Kawano, Ryoko Ando, Tomomi Shimogori, Hisayori Noda, Kiyoko Fukami, Asako Sakaue-Sawano, and Atsushi Miyawaki. Scale: a chemical approach for fluorescence imaging and reconstruction of transparent mouse brain. Nat Neurosci, 14(11):1481-8, Nov 2011.

[6] Meng-Tsen Ke, Satoshi Fujimoto, and Takeshi Imai. Seedb: a simple and morphology-preserving optical clearing agent for neuronal circuit reconstruction. Nat Neurosci, 16(8):1154-61, Aug 2013.

[7] Etsuo A Susaki, Kazuki Tainaka, Dimitri Perrin, Fumiaki Kishino, Takehiro Tawara, Tomonobu M Watanabe, Chihiro Yokoyama, Hirotaka Onoe, Megumi Eguchi, Shun Yamaguchi, Takaya Abe, Hiroshi Kiyonari, Yoshihiro Shimizu, Atsushi Miyawaki, Hideo Yokota, and Hiroki R Ueda. Whole-brain imaging with single-cell resolution using chemical cocktails and computational analysis. Cell, 157(3):726-39, Apr 2014.

[8] Gregory S X E Jefferis, Christopher J Potter, Alexander M Chan, Elizabeth C Marin, Torsten Rohlfing, Calvin R Maurer, and Liqun Luo. Comprehensive maps of drosophila higher olfactory centers: spatially segregated fruit and pheromone representation. Cell, 128(6):1187-203, Mar 2007.

[9] Sebastian Cachero, Aaron D Ostrovsky, Jai Y Yu, Barry J Dickson, and Gregory S X E Jefferis. Sexual dimorphism in the fly brain. Curr Biol, Sep 2010.

[10] Jai Y Yu, Makoto I Kanai, Ebru Demir, Gregory S X E Jefferis, and Barry J Dickson. Cellular organization of the neural circuit that drives drosophila courtship behavior. Curr Biol, 20(18):1602-14, Sep 2010.

[11] Ann-Shyn Chiang, Chih-Yung Lin, Chao-Chun Chuang, Hsiu-Ming Chang, Chang-Huain Hsieh, ChangWei Yeh, Chi-Tin Shih, Jian-Jheng Wu, Guo-Tzau Wang, Yung-Chang Chen, Cheng-Chi Wu, Guan-Yu Chen, Yu-Tai Ching, Ping-Chang Lee, Chih-Yang Lin, Hui-Hao Lin, Chia-Chou Wu, Hao-Wei Hsu, YunAnn Huang, Jing-Yi Chen, Hsin-Jung Chiang, Chun-Fang Lu, Ru-Fen Ni, Chao-Yuan Yeh, and JennKang Hwang. Three-dimensional reconstruction of brain-wide wiring networks in drosophila at single-cell resolution. Curr Biol, 21(1):1-11, Jan 2011.

[12] Arnim Jenett, Gerald M Rubin, Teri-T B Ngo, David Shepherd, Christine Murphy, Heather Dionne, Barret D Pfeiffer, Amanda Cavallaro, Donald Hall, Jennifer Jeter, Nirmala Iyer, Dona Fetter, Joanna H Hausenfluck, Hanchuan Peng, Eric T Trautman, Robert R Svirskas, Eugene W Myers, Zbigniew R Iwinski, Yoshinori Aso, Gina M DePasquale, Adrianne Enos, Phuson Hulamm, Shing Chun Benny Lam, Hsing-Hsi Li, Todd R Laverty, Fuhui Long, Lei Qu, Sean D Murphy, Konrad Rokicki, Todd Safford, Kshiti Shaw, Julie H Simpson, Allison Sowell, Susana Tae, Yang Yu, and Christopher T Zugates. A gal4-driver line resource for drosophila neurobiology. Cell Rep, 2(4):991-1001, Oct 2012.

[13] Johannes Kohl, Aaron D Ostrovsky, Shahar Frechter, and Gregory S X E Jefferis. A bidirectional circuit switch reroutes pheromone signals in male and female brains. Cell, 155(7):1610-23, Dec 2013. 
[14] Joy S Wu and Liqun Luo. A protocol for dissecting drosophila melanogaster brains for live imaging or immunostaining. Nat Protoc, 1(4):2110-5, 2006.

[15] Antje Keppler, Susanne Gendreizig, Thomas Gronemeyer, Horst Pick, Horst Vogel, and Kai Johnsson. A general method for the covalent labeling of fusion proteins with small molecules in vivo. Nat Biotechnol, 21(1):86-9, Jan 2003.

[16] Arnaud Gautier, Alexandre Juillerat, Christian Heinis, Ivan Reis Corrêa, Jr, Maik Kindermann, Florent Beaufils, and Kai Johnsson. An engineered protein tag for multiprotein labeling in living cells. Chem Biol, 15(2):128-36, Feb 2008.

[17] Xiaoli Sun, Aihua Zhang, Brenda Baker, Luo Sun, Angela Howard, John Buswell, Damien Maurel, Anastasiya Masharina, Kai Johnsson, Christopher J Noren, Ming-Qun Xu, and Ivan R Corrêa, Jr. Development of snap-tag fluorogenic probes for wash-free fluorescence imaging. Chembiochem, 12(14):2217-26, Sep 2011.

[18] Patrina A Pellett, Xiaoli Sun, Travis J Gould, James E Rothman, Ming-Qun Xu, Ivan R Corrêa, Jr, and Joerg Bewersdorf. Two-color sted microscopy in living cells. Biomed Opt Express, 2(8):2364-71, Aug 2011.

[19] Georgyi V Los, Lance P Encell, Mark G McDougall, Danette D Hartzell, Natasha Karassina, Chad Zimprich, Monika G Wood, Randy Learish, Rachel Friedman Ohana, Marjeta Urh, Dan Simpson, Jacqui Mendez, Kris Zimmerman, Paul Otto, Gediminas Vidugiris, Ji Zhu, Aldis Darzins, Dieter H Klaubert, Robert F Bulleit, and Keith V Wood. Halotag: a novel protein labeling technology for cell imaging and protein analysis. ACS Chem Biol, 3(6):373-82, Jun 2008.

[20] Lance P Encell, Rachel Friedman Ohana, Kris Zimmerman, Paul Otto, Gediminas Vidugiris, Monika G Wood, Georgyi V Los, Mark G McDougall, Chad Zimprich, Natasha Karassina, Randall D Learish, Robin Hurst, James Hartnett, Sarah Wheeler, Pete Stecha, Jami English, Kate Zhao, Jacqui Mendez, Hélène A Benink, Nancy Murphy, Danette L Daniels, Michael R Slater, Marjeta Urh, Aldis Darzins, Dieter H Klaubert, Robert F Bulleit, and Keith V Wood. Development of a dehalogenase-based protein fusion tag capable of rapid, selective and covalent attachment to customizable ligands. Curr Chem Genomics, 6:55-71, 2012.

[21] Lawrence W Miller, Yunfei Cai, Michael P Sheetz, and Virginia W Cornish. In vivo protein labeling with trimethoprim conjugates: a flexible chemical tag. Nat Methods, 2(4):255-7, Apr 2005.

[22] Sarah S Gallagher, Julia E Sable, Michael P Sheetz, and Virginia W Cornish. An in vivo covalent tmp-tag based on proximity-induced reactivity. ACS Chem Biol, 4(7):547-56, Jul 2009.

[23] Zhixing Chen, Chaoran Jing, Sarah S Gallagher, Michael P Sheetz, and Virginia W Cornish. Secondgeneration covalent tmp-tag for live cell imaging. J Am Chem Soc, 134(33):13692-9, Aug 2012.

[24] Marlon J Hinner and Kai Johnsson. How to obtain labeled proteins and what to do with them. Curr Opin Biotechnol, 21(6):766-76, Dec 2010.

[25] A H Brand and N Perrimon. Targeted gene expression as a means of altering cell fates and generating dominant phenotypes. Development, 118(2):401-15, Jun 1993.

[26] Ebru Demir and Barry J Dickson. fruitless splicing specifies male courtship behavior in drosophila. Cell, 121(5):785-94, Jun 2005.

[27] G S Jefferis, E C Marin, R F Stocker, and L Luo. Target neuron prespecification in the olfactory map of drosophila. Nature, 414(6860):204-8, Nov 2001. 
[28] Gregory S X E Jefferis, Raj M Vyas, Daniela Berdnik, Ariane Ramaekers, Reinhard F Stocker, Nobuaki K Tanaka, Kei Ito, and Liqun Luo. Developmental origin of wiring specificity in the olfactory system of drosophila. Development, 131(1):117-30, Jan 2004.

[29] Yong Q Zhang, Christopher K Rodesch, and Kendal Broadie. Living synaptic vesicle marker: synaptotagmin-gfp. Genesis, 34(1-2):142-5, 2002.

[30] Ya-Hui Chou, Maria L Spletter, Emre Yaksi, Jonathan C S Leong, Rachel I Wilson, and Liqun Luo. Diversity and wiring variability of olfactory local interneurons in the drosophila antennal lobe. Nat Neurosci, 13(4):439-49, Apr 2010.

[31] Laura J J Nicolaï, Ariane Ramaekers, Tim Raemaekers, Andrzej Drozdzecki, Alex S Mauss, Jiekun Yan, Matthias Landgraf, Wim Annaert, and Bassem A Hassan. Genetically encoded dendritic marker sheds light on neuronal connectivity in drosophila. Proc Natl Acad Sci U S A, 107(47):20553-8, Nov 2010.

[32] Dafni Hadjieconomou, Shay Rotkopf, Cyrille Alexandre, Donald M Bell, Barry J Dickson, and Iris Salecker. Flybow: genetic multicolor cell labeling for neural circuit analysis in drosophila melanogaster. Nat Methods, 8(3):260-6, Mar 2011.

[33] Dawen Cai, Kimberly B Cohen, Tuanlian Luo, Jeff W Lichtman, and Joshua R Sanes. Improved tools for the brainbow toolbox. Nat Methods, 10(6):540-7, May 2013.

[34] Robert J Kittel, Carolin Wichmann, Tobias M Rasse, Wernher Fouquet, Manuela Schmidt, Andreas Schmid, Dhananjay A Wagh, Christian Pawlu, Robert R Kellner, Katrin I Willig, Stefan W Hell, Erich Buchner, Manfred Heckmann, and Stephan J Sigrist. Bruchpilot promotes active zone assembly, ca2+ channel clustering, and vesicle release. Science, 312(5776):1051-4, May 2006.

[35] Karlheinz Rein, Malte Zöckler, Michael T Mader, Cornelia Grübel, and Martin Heisenberg. The drosophila standard brain. Curr Biol, 12(3):227-31, Feb 2002.

[36] Nestor Milyaev, David Osumi-Sutherland, Simon Reeve, Nicholas Burton, Richard A Baldock, and J Douglas Armstrong. The virtual fly brain browser and query interface. Bioinformatics, 28(3):411-5, Feb 2012.

[37] Kei Ito, Kazunori Shinomiya, Masayoshi Ito, J Douglas Armstrong, George Boyan, Volker Hartenstein, Steffen Harzsch, Martin Heisenberg, Uwe Homberg, Arnim Jenett, Haig Keshishian, Linda L Restifo, Wolfgang Rössler, Julie H Simpson, Nicholas J Strausfeld, Roland Strauss, Leslie B Vosshall, and Insect Brain Name Working Group. A systematic nomenclature for the insect brain. Neuron, 81(4):755-65, Feb 2014.

[38] Gregory S X E Jefferis, Christopher J Potter, Alexander M Chan, Elizabeth C Marin, Torsten Rohlfing, Calvin R Maurer, Jr, and Liqun Luo. Comprehensive maps of drosophila higher olfactory centers: spatially segregated fruit and pheromone representation. Cell, 128(6):1187-203, Mar 2007.

[39] Sandeep Robert Datta, Maria Luisa Vasconcelos, Vanessa Ruta, Sean Luo, Allan Wong, Ebru Demir, Jorge Flores, Karen Balonze, Barry J Dickson, and Richard Axel. The drosophila pheromone cva activates a sexually dimorphic neural circuit. Nature, 452(7186):473-477, Mar 2008.

[40] Vanessa Ruta, Sandeep Robert Datta, Maria Luisa Vasconcelos, Jessica Freeland, Loren L Looger, and Richard Axel. A dimorphic pheromone circuit in drosophila from sensory input to descending output. Nature, 468(7324):686-90, Dec 2010.

[41] Masayoshi Ito, Naoki Masuda, Kazunori Shinomiya, Keita Endo, and Kei Ito. Systematic analysis of neural projections reveals clonal composition of the drosophila brain. Curr Biol, 23(8):644-55, Apr 2013. 
[42] Hung-Hsiang Yu, Takeshi Awasaki, Mark David Schroeder, Fuhui Long, Jacob S Yang, Yisheng He, Peng Ding, Jui-Chun Kao, Gloria Yueh-Yi Wu, Hanchuan Peng, Gene Myers, and Tzumin Lee. Clonal development and organization of the adult drosophila central brain. Curr Biol, Mar 2013.

[43] Mamie Z Li and Stephen J Elledge. Harnessing homologous recombination in vitro to generate recombinant dna via slic. Nat Methods, 4(3):251-6, Mar 2007.

[44] Daniel G Gibson, Lei Young, Ray-Yuan Chuang, J Craig Venter, Clyde A Hutchison, 3rd, and Hamilton O Smith. Enzymatic assembly of dna molecules up to several hundred kilobases. Nat Methods, 6(5):343-5, May 2009.

[45] Jiayuan Quan and Jingdong Tian. Circular polymerase extension cloning of complex gene libraries and pathways. PLoS One, 4(7):e6441, 2009.

[46] Yongwei Zhang, Uwe Werling, and Winfried Edelmann. Slice: a novel bacterial cell extract-based dna cloning method. Nucleic Acids Res, 40(8):e55, Apr 2012.

[47] Shu Kondo and Ryu Ueda. Highly improved gene targeting by germline-specific cas9 expression in drosophila. Genetics, 195(3):715-21, Nov 2013.

[48] Andrew R Bassett, Charlotte Tibbit, Chris P Ponting, and Ji-Long Liu. Highly efficient targeted mutagenesis of drosophila with the crispr/cas9 system. Cell Rep, 4(1):220-8, Jul 2013.

[49] Scott J Gratz, Alexander M Cummings, Jennifer N Nguyen, Danielle C Hamm, Laura K Donohue, Melissa M Harrison, Jill Wildonger, and Kate M O'Connor-Giles. Genome engineering of drosophila with the crispr rna-guided cas9 nuclease. Genetics, 194(4):1029-35, Aug 2013.

[50] Zhongsheng Yu, Mengda Ren, Zhanxiang Wang, Bo Zhang, Yikang S Rong, Renjie Jiao, and Guanjun Gao. Highly efficient genome modifications mediated by crispr/cas9 in drosophila. Genetics, 195(1):28991, Sep 2013.

[51] Xingjie Ren, Jin Sun, Benjamin E Housden, Yanhui Hu, Charles Roesel, Shuailiang Lin, Lu-Ping Liu, Zhihao Yang, Decai Mao, Lingzhu Sun, Qujie Wu, Jun-Yuan Ji, Jianzhong Xi, Stephanie E Mohr, Jiang $\mathrm{Xu}$, Norbert Perrimon, and Jian-Quan Ni. Optimized gene editing technology for drosophila melanogaster using germ line-specific cas9. Proc Natl Acad Sci U S A, 110(47):19012-7, Nov 2013.

[52] Woong Y Hwang, Yanfang Fu, Deepak Reyon, Morgan L Maeder, Shengdar Q Tsai, Jeffry D Sander, Randall T Peterson, J-R Joanna Yeh, and J Keith Joung. Efficient genome editing in zebrafish using a crispr-cas system. Nat Biotechnol, 31(3):227-9, Mar 2013.

[53] Hui Yang, Haoyi Wang, Chikdu S Shivalila, Albert W Cheng, Linyu Shi, and Rudolf Jaenisch. One-step generation of mice carrying reporter and conditional alleles by crispr/cas-mediated genome engineering. Cell, 154(6):1370-9, Sep 2013.

[54] Nathalie George, Horst Pick, Horst Vogel, Nils Johnsson, and Kai Johnsson. Specific labeling of cell surface proteins with chemically diverse compounds. J Am Chem Soc, 126(29):8896-7, Jul 2004.

[55] Jean Livet, Tamily A Weissman, Hyuno Kang, Ryan W Draft, Ju Lu, Robyn A Bennis, Joshua R Sanes, and Jeff W Lichtman. Transgenic strategies for combinatorial expression of fluorescent proteins in the nervous system. Nature, 450(7166):56-62, Nov 2007.

[56] Stefanie Hampel, Phuong Chung, Claire E McKellar, Donald Hall, Loren L Looger, and Julie H Simpson. Drosophila brainbow: a recombinase-based fluorescence labeling technique to subdivide neural expression patterns. Nat Methods, 8(3):253-9, Mar 2011. 
[57] R. F. Stocker, G. Heimbeck, N. Gendre, and J. S. de Belle. Neuroblast ablation in drosophila p[gal4] lines reveals origins of olfactory interneurons. J. Neurobio., 32:443-452, 1997.

[58] Barret D Pfeiffer, Teri-T B Ngo, Karen L Hibbard, Christine Murphy, Arnim Jenett, James W Truman, and Gerald M Rubin. Refinement of tools for targeted gene expression in drosophila. Genetics, 186(2):735-55, Oct 2010 .

[59] Michael D Gordon and Kristin Scott. Motor control in a drosophila taste circuit. Neuron, 61(3):373-84, Feb 2009.

[60] Barret D Pfeiffer, James W Truman, and Gerald M Rubin. Using translational enhancers to increase transgene expression in drosophila. Proc Natl Acad Sci U S A, 109(17):6626-31, Apr 2012.

[61] Koen J T Venken, Karen L Schulze, Nele A Haelterman, Hongling Pan, Yuchun He, Martha Evans-Holm, Joseph W Carlson, Robert W Levis, Allan C Spradling, Roger A Hoskins, and Hugo J Bellen. Mimic: a highly versatile transposon insertion resource for engineering drosophila melanogaster genes. Nat Methods, 8(9):737-43, Sep 2011.

[62] Dhananjay A Wagh, Tobias M Rasse, Esther Asan, Alois Hofbauer, Isabell Schwenkert, Heike Dürrbeck, Sigrid Buchner, Marie-Christine Dabauvalle, Manuela Schmidt, Gang Qin, Carolin Wichmann, Robert Kittel, Stephan J Sigrist, and Erich Buchner. Bruchpilot, a protein with homology to elks/cast, is required for structural integrity and function of synaptic active zones in drosophila. Neuron, 49(6):833-44, Mar 2006.

[63] Sophie J C Caron, Vanessa Ruta, L F Abbott, and Richard Axel. Random convergence of olfactory inputs in the drosophila mushroom body. Nature, 497(7447):113-7, May 2013. 


\section{ONLINE METHODS}

\section{Drosophila stocks}

All flies used in this study were 2-4 d old males with the exception of PA-GFP tracing experiments (see below). fruitless ${ }^{\text {Gal4 }}\left(\right.$ fru $\left.^{\text {Gal4 }}\right)$ is a targeted insertion of the yeast transcription factor Gal4 into the P1 promoter of the fruitless gene ${ }^{26}$. GH146-Gal4 ${ }^{57}$ and Mz19-Gal4 ${ }^{28}$ are $\mathrm{P}$ element enhancer trap insertions. The generation of constructs for transgenic fly stocks is described below. UAS-DenMark ${ }^{31}$, Syt-EGFP ${ }^{29}$, syb-EGFP ${ }^{29}$ and $m y r-G F P^{58}$ flies were obtained from Bloomington Stock Center.

\section{Drosophila constructs}

- pTW<Syt-CLIPm>, pTW<Syt-SNAPm>, pTW $<$ CLIPf-Syb $>$ and pTW $<$ SNAPf-Syb $>$ constructs were made using the Multisite Gateway technology platform (Invitrogen). CLIPf and SNAPf are engineered versions of the original versions CLIP26m (CLIPm) and SNAP26m (SNAPm), respectively, that display faster labeling kinetics ${ }^{17,18}$. pTW is a pUASt vector containing a Gateway cassette. All CLIPm, CLIPf, SNAPm and SNAPf coding sequences were amplified from pCLIPm, pCLIPf, pSNAPm or pSNAPf plamids (NEB), respectively. Drosophila synaptotagmin 1 (syt1, GenBank M55048) was amplified from $P\{U A S$-syt.eGFP $\} 1$ flies (Bloomington, ${ }^{29}$ ) using syt forward and EGFP reverse primers. Drosophila $n$-synaptobrevin (n-syb, GenBank S66686) was amplified from $P\{U A S$ - $n$-syb.eGFP $\} 2$ flies using syb forward and EGFP reverse primers. All synaptotagmin constructs described in this study are C-terminal, i.e. cytoplasmic fusions. All synaptobrevin constructs described here are $\mathrm{N}$-terminal fusions, i.e. cytoplasmic.

- UAS-TLN-CLIPm and UAS-TLN-SNAPm constructs were generated as follows: First, fusion PCR was performed on a pUAST-TLN-cherry plasmid to remove the mCherry coding sequence and flanking linker sequences (pUAST-TLN $\Delta$ cherry). A $1.5 \mathrm{~kb}$ fragment from the XhoI restriction site to the sequence corresponding to amino acids TVRVA of mouse Telencephalin/ICAM-5 and a $1.0 \mathrm{~kb}$ fragment ranging from amino acids GPWLW of Telencephalin/ICAM-5 to the MfeI restriction site were amplified. Using primers that introduced flanking XhoI and BgIII sites, the full $2.7 \mathrm{~kb}$ fragment was cloned into pUAST-TLN $\Delta$ cherry (pUAST-TLN). CLIPm and SNAPm sequences were amplified using BglIIcontaining primers and inserted into pUAST-TLN, thus yielding pUAST-TLN-CLIP and pUAST-TLNSNAP.

- pTW $<$ PAT3SP-CD4-CLIPf $>$ and pTW $<$ PAT3SP-CD4-SNAPf $>$ constructs were made using the Multisite Gateway technology platform. PAT3 signal peptide (SP) and CD4 sequences were amplified from UASCD4::spGFP1-10 flies $^{59}$.

- The UAS-myr-SNAPf construct was generated as follows: the GFP coding sequence was removed from a pJFRC-MUH-myr-GFP construct ${ }^{58}$ using BamHI/KpnI restriction sites and the SNAPf coding sequence was amplified from pSNAPf (NEB) and inserted.

- The UAS-myr-TMP construct was generated as follows: the GFP coding sequence was excised from a pJFRC81-L21 construct ${ }^{60}$ using BamHI/KpnI restriction sites and replaced with the eDHFR coding sequence (codon-optimized for Drosophila) and an N-terminal myristoylation signal.

- The UAS-myr-Halo construct was generated as follows: the TMP coding sequence was excised from UAS-myr-TMP (see above) using BamHI/KpnI restriction sites and replaced with a Halo-tag sequence amplified from the pHT2 Halo-tag plasmid (Promega). 


\section{Generation of brp-SNAP}

We targeted the bruchpilot (brp) gene taking advantage of a MiMIC ${ }^{61}$ insertion (MI02987) $6.8 \mathrm{~kb}$ upstream of the stop codon present in the last coding exon of brp (Fig. S4). Because this exon is common to all nine Brp isoforms annotated in Flybase, we anticipated that inserting the SNAP ORF immediately 5' to the stop codon would label all Brp isoforms. The brp-SNAP targeting construct was generated by Gibson assembly ${ }^{44}$ of the following fragments into the backbone of a pDONR221 plasmid.

- three fragments encompassing the DNA sequence from the MiMIC insertion site to the end of the last coding exon

- SNAP coding sequence (added 5' to the stop codon of brp)

- a $2.2 \mathrm{~kb}$ fragment of the brp 3' UTR and a cleavage site for the homing endonuclease I-CreI

- a fragment containing the 3xP3 promoter driving expression of RFP (used for screening of successful reduction step (see below).

The entire assembly was flanked by PhiC31 sites on both sides. Transgenic flies with the brp-SNAP construct replacing the original MiMIC insertion were made (BestGene Inc.) and genotyped to verify the landing site and orientation of the integration. Two positive lines were identified and assayed by anti-SNAP staining. Since the integration into the brp locus generates a large duplication and an incomplete 3' UTR (Fig. S4), one positive line was crossed to a hs-I-CreI line. The progeny were heat shocked for $30 \mathrm{~min}$ and subsequently crossed to balancers. Progeny from this cross were then screened for loss of the RFP eye marker, indicating successful repair of the I-CreI induced double strand break, resulting in reduction of the duplication and restoration of the 3' UTR. One such fly was identified and used to generate a stock wich was then verified by PCR (Fig. S4d) and anti-SNAP staining.

\section{Chemical stainings and immunohistochemistry}

Labeling reagents SNAP- and CLIP-Surface (i.e. cell-impermeable) labeling substrates are abbreviated as BG- (benzylguanine) or BC- (benzylcytosine) in this study (see Table S2 for all abbreviations). Substrates were either acquired as stock solutions (e.g. Halo-TMR) or acquired in powder form and dissolved in fresh, water-free dimethyl sulfoxide (DMSO, Sigma) at a concentration of $1 \mathrm{mM}$ (SNAP and CLIP substrates). Aliquots $(5 \mu \mathrm{l})$ were stored at $-20{ }^{\circ} \mathrm{C}$ in presence of desiccant. We observed that using old DMSO or storing dissolved substrates in wet and/or warm conditions can lead to hydrolysis, drastically reducing labeling efficiency (see 'Protocols for chemical labeling' below).

Fly brains Immunohistochemistry was carried out as follows: brains were dissected in $0.1 \mathrm{M}$ phosphate buffer (PB), then fixed in 4\% paraformaldehyde (PFA, Electron Microscopy Services, Hatfield, PA, USA) in PB at room temperature for $20 \mathrm{~min}$ (see ref $\left.\left[{ }^{8}\right]\right)$. We observed that prolonged fixation $(1 \mathrm{~h}$ at room temperature) improved signal intensity for some far-red SNAP substrates (e.g. BG-TF5), while decreasing labeling of Halotag. For antibody stainings and non cell-permeable substrates, brains were then permeabilized by washing in PBT (phosphate-buffered saline $+0.3 \%$ Triton, $2 \times 5 \mathrm{~min}$ ). Cell-permeable substrates were added without permeabilizing the brains. For immunostainings, blocking with 5\% goat serum was performed overnight at $4{ }^{\circ} \mathrm{C}$. No blocking was performed for chemical stainings. All following washing steps were performed in PBT (immunostainings and chemical stainings with non cell-permeable substrates) or PBS (cell-permeable substrates) for $2 \times 10 \mathrm{~min}$. Fluorescent substrates (see Table S2) were added at a concentration of $0.1-1$ $\mu \mathrm{M}$ for $1 \mathrm{~min}-12 \mathrm{~h}$ (on a rotating wheel, at room temperature for incubations $<4 \mathrm{~h}$, at $4{ }^{\circ} \mathrm{C}$ for incubations $>4 \mathrm{~h}$ ). An increase in background signal was typically observed for incubations of cell-permeable substrates for $>2 \mathrm{~h}$. For immunostainings, prolonged incubation of 2 days each with primary and secondary antibodies (rotating at $4{ }^{\circ} \mathrm{C}$ ) was required for homogeneous staining. Primary antibodies were: mouse anti-nc $82^{62}$ (DSHB, 
University of Iowa) 1:20-1:40, chicken anti-GFP (Abcam, ab13970) 1:1000, rat anti-HA (Roche, 11867423 001) 1:200. Secondary antibodies (all from Life Technologies) were: Alexa-568 anti-mouse (A-11004) 1:1200, Alexa-633 anti-mouse (A-21052) 1:1200, Alexa-488 anti-chicken (A-11039) 1:1200, Alexa-568 anti-rabbit (A11011) 1:1200. Specimens were whole mounted in Vectashield (Vector Laboratories) on charged slides to avoid movement.

\section{Image acquisition}

Confocal stacks of fly brains were acquired using a Zeiss 710 confocal microscope. Brains were imaged at 768 $\times 768$ pixel resolution every $1 \mu \mathrm{m}$ (voxel size $0.46 \times 0.46 \times 1 \mu \mathrm{m}$ ) using an EC Plan-Neofluar $40 \times / 1.30$ Oil objective and 0.6 zoom factor. Images of dye-filled neurons were acquired with $2 \times$ (frame) averaging. Detail images were taken with a Plan-Apochromat $63 \times / 1.4$ Oil objective at $2-3 \times$ zoom and contained about 30 slices (768 $\times 768$ pixels) with a voxel size of $0.06 \times 0.06 \times 0.15 \mu \mathrm{m}$. All images were taken using 16 -bit color depths. Confocal images were registered to the IS 2 template brain as previously described ${ }^{9}$ using the Computational Morphometry Toolkit (CMTK) available at http://www.nitrc.org/projects/cmtk.

\section{PA-GFP tracing and brp-SNAP labeling}

Brains from 1-2 day old flies were dissected in ice-cold artificial hemolymph $(108 \mathrm{mM} \mathrm{NaCl}, 5 \mathrm{mM} \mathrm{KCl}$, $2 \mathrm{mM} \mathrm{CaCl}_{2}, 8.2 \mathrm{mM} \mathrm{MgCl}_{2}, 4 \mathrm{mM} \mathrm{NaHCO}, 1 \mathrm{mM} \mathrm{NaH}_{2} \mathrm{PO}_{4}, 5 \mathrm{mM}$ trehalose, $10 \mathrm{mM}$ sucrose, $5 \mathrm{mM}$ HEPES, pH 7.5, $265 \mathrm{mOsm}$ ) and allowed to adhere to a Poly-d-lysine treated coverslip within a cell culture dish. Photoactivation was performed with a two-photon microscope as described ${ }^{63}$. Briefly, several Kenyon cells were selected for photoactivation using baseline fluorescence at $925 \mathrm{~nm}$. The somata of these cells were continuously illuminated with $710 \mathrm{~nm}$ light for $2 \mathrm{~min}$, followed by a 30 min resting period to allow for diffusion of photoactivated GFP. The brain was then fixed in 4\% PFA in PB for $20 \mathrm{~min}$ at room temperature and washed in PBT $(3 \times)$. Subsequently, $200 \mathrm{nM}$ of BG-549 substrate in PBT were added for $20 \mathrm{~min}$. Finally, brains were briefly washed with PBT, equilibrated in Vectashield, mounted and imaged.

\section{Image analysis and quantification of labeling}

For quantification of substrate penetration into fly brains, samples from GH146-Gal4 > myrSNAP or GH146Gal4 > myrHalo or brp-SNAP animals were incubated with $1 \mu \mathrm{M}$ BG-549 or $1 \mu \mathrm{M}$ Halo-TMR for $15 \mathrm{~min}, 30$ min, $1 \mathrm{~h}, 4 \mathrm{~h}$ or $12 \mathrm{~h}$. Samples from GH146-Gal4 > myrGFP or Canton-S animals were incubated with anti-GFP $(1: 1,000)$ or anti-nc82 (1:30) antibodies for $15 \mathrm{~min}, 30 \mathrm{~min}, 1 \mathrm{~h}, 4 \mathrm{~h}$ or $12 \mathrm{~h}$, followed by washing and incubation with fluorescent secondary antibodies (anti-chicken Alexa 568 and anti-mouse Alexa 568, respectively) for either $2 \mathrm{~d}$ or for the same time as primary antibodies (i.e. $15 \mathrm{~min}, 30 \mathrm{~min}, 1 \mathrm{~h}, 4 \mathrm{~h}$ or $12 \mathrm{~h}$ ).

Quantification of GH146 labeling All 3D confocal stacks were registered onto a GH146-Gal4 > myrGFP template. GH146-positive areas were segmented from each registered brain using a binary mask (surfaced rendered in Fig. S7b) constructed as follows: five GH146-Gal4 > myrGFP brains (stained with anti-GFP antibody for $12 \mathrm{~h}$ ) were averaged in Fiji. The resulting image stack was filtered (median + Gaussian) and thresholded.

Quantification of brp-SNAP/nc82 labeling All images were registered against an nc82 template brain $\left(\right.$ IS $2^{9}$ ). Two binary masks were made in order to separate neuropil and cortex (see Fig. S7a): five brp-SNAP brains labeled with BG-549 were averaged, filtered (median + Gaussian) and thresholded. In order to assess cortical background labeling, signal was quantified in the region resulting from subtraction of the neuropil mask from the whole brain mask (see Fig. S7a). 


\section{Protocol for chemical labeling of Drosophila brains}

- dissect brains in ice-cold 0.1 M PB

- fixation in 4\% PFA (in $0.1 \mathrm{M} \mathrm{PB}$ ) at room temperature for 20 min in glass-well plate on nutator

- SNAP-tag / CLIP-tag: in general no change in labeling intensity after prolonged fixation of up to 60 min however, we observed improved labeling intensity for BG-649 substrate after 60 min fixation.

- Halo-tag: labeling intensity significantly decreased with longer fixation (40-60 min)

- TMP-tag: labeling intensity significantly increased with longer fixation (40-60 min)

- (1) non cell-permeable substrates:

- transfer brains to $1.5 \mathrm{ml}$ tube, permeabilize briefly (5-10 $\mathrm{min}$ ) by incubation in $0.5 \mathrm{ml}$ of PBT ( $0.3 \%$ Triton X-100) on rotating wheel

- add substrate for a final concentration of $1 \mu \mathrm{M}$ in PBT (see notes on substrates below) higher concentrations can result in higher background

- incubate brains on rotating wheel for 15 min agitation of the sample(s) is important to obtain rapid, homogeneous staining! long incubation times ( $>6 \mathrm{~h}$ ) can result in increased background staining!

- wash brains with PBT $(2 \times 10 \mathrm{~min})$

- (2) cell-permeable substrates:

- transfer brains to $1.5 \mathrm{ml}$ tube, wash briefly (5-10 $\mathrm{min})$ in PBS

- add substrate for a final concentration of $1-5 \mu \mathrm{M}$ in PBS higher concentrations usually result in considerable background; using cell-permeable substrates in presence of Triton typically results in very weak labeling (when using cell-permeable substrates on permeabilized samples, wash sample with PBS before adding substrate

- incubate brains on rotating wheel for 15 min agitation of the sample(s) is important to obtain rapid, homogeneous staining! long incubation times $(>6 \mathrm{~h}$ ) can result in slightly increased background staining!

- wash brains with PBS, or - to reduce background - with PBT

- remove PBT as completely as possible and add $\sim 200 \mu$ of Vectashield (or other) mounting medium we observed that subsequently transferring brains into a fresh $\sim 200 \mu$ aliquot of Vectashield results in more homogeneous signal along $z$

- mount brains on charged slides and image 


\section{Notes on substrates for chemical labeling}

- store substrates at $-20{ }^{\circ} \mathrm{C}$ in presence of dessicant this avoids hydrolysis of substrates

- for substrates that are purchased in dry powder form: dissolve in fresh, water-free DMSO using fresh DMSO from a sealed container is crucial as DMSO is hygroscopic; we observed reduced labeling efficiency when using substrates that were dissolved in old DMSO, presumably due to substrate hydrolysis

- avoid freeze-thawing, aliquot samples (e.g. $2 \mu \mathrm{l}$ aliquots in $0.2 \mathrm{ml}$ PCR tubes) we observed a decrease in labeling efficiency for some substrates that underwent several freeze-thaw cycles (e.g. BG-649)

- BG-488, BG-549, BG-649, BC-547, BC-488, Halo-TMR and TMP-Fluorescein work well using our standard staining protocol

- when using chemical labeling in combination with immunostainings, substrates should be added right after tissue permeabilization (not after the o/n blocking step) to obtain optimal results

- Cost of chemical labeling: the cost of $50 \mathrm{nmol}$ of SNAP- or CLIP-substrate (sufficient for 1,000 labeling reactions at $1 \mu \mathrm{M}$ concentration in a volume of $0.5 \mathrm{ml}$ each) is comparable to that of an $0.5 \mathrm{ml}$ aliquot of fluorophore-coupled secondary antibody (sufficient for $\sim 1,000$ immunostainings in a volume of $0.5 \mathrm{ml}$ each). However, we have successfully tested some substrates (e.g. BG-549, Halo-TMR) at $100 \mathrm{nM}$ and lower concentrations (indeed, there might be situations where lower substrate concentrations - combined with longer incubation times - might be desirable). Also, no primary antibodies and blocking reagents are required for chemical labeling. Therefore, the cost of chemical labeling is comparable to, or even lower than, the cost of immunostainings. 


\section{ACKNOWLEDGMENTS}

We thank K. Johnsson, V. Cornish and I. Correa for providing labeling reagents, A. Carter and B. Hassan for DNA constructs, the Developmental Studies Hybridoma Bank for antibodies, and the Bloomington Stock Center for fly stocks. We thank I. Correa for helpful discussions and L. Luo, M. Landgraf, A. Ostrovsky, A. Jenett and members of the Jefferis group for comments on the manuscript. This study made use of the Computational Morphometry Toolkit, supported by the National Institute of Biomedical Imaging and Bioengineering. This work was supported by a European Research Council Starting Investigator Grant, the Medical Research Council (MRC file reference MC_U105188491) and the EMBO Young Investigator Program (GSXEJ); an MRC LMB Graduate Scholarship (JK); a Boehringer Ingelheim Fonds predoctoral fellowship (MJD); a Royal Society Dorothy Hodgkin Fellowship (SC) and an EMBO post-doctoral fellowship (SF).

\section{AUTHOR CONTRIBUTIONS}

JK and GSXEJ initiated the project. JK, JN, SC and GSXEJ designed the experiments. JK, JN, SC and DK made constructs and/or transgenic flies. JK, JN, SC, MJD, BS and SF carried out experiments. JK and GSXEJ analyzed data. JK, SC and GSXEJ prepared figures. JK, JN and GSXEJ wrote the manuscript with input from all authors. 


\section{FIGURES}

\section{List of Figures}

1 Expression of chemical tags in the fly brain . . . . . . . . . . . . . . . . . . . . . . . 19

2 Ultrafast and homogeneous tag-based tissue staining . . . . . . . . . . . . . . . . . . . . 20

3 Key applications for chemical labeling in Drosophila neurobiology . . . . . . . . . . . . . . . . . . . . . . . . 22

S1 Additional chemical tag reporter fly lines . . . . . . . . . . . . . . . . . . . . . . . . .

S2 Cross-reactivity of tag-substrate combinations used in this study . . . . . . . . . . . . . . . . . 6

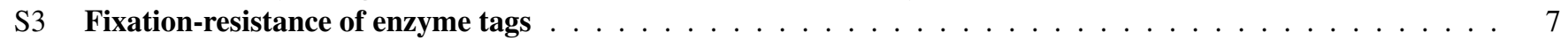

S4 Generation of $b r p-S N A P \ldots \ldots \ldots$

S5 Comparison of neuropil antibody staining and labeling using the brp-SNAP transgene . . . . . . . . . . . 9

S6 Sequential labeling reveals binding kinetics . . . . . . . . . . . . . . . . . . . . . . 10

S7 Masks used for quantification of labeling intensity in fly brains . . . . . . . . . . . . . . . . . . . . . . . . 11 
bioRxiv preprint doi: https://doi.org/10.1101/005298; this version posted May 19, 2014. The copyright holder for this preprint (which was not certified by peer review) is the author/funder, who has granted bioRxiv a license to display the preprint in perpetuity. It is made available under aCC-BY-ND 4.0 International license.
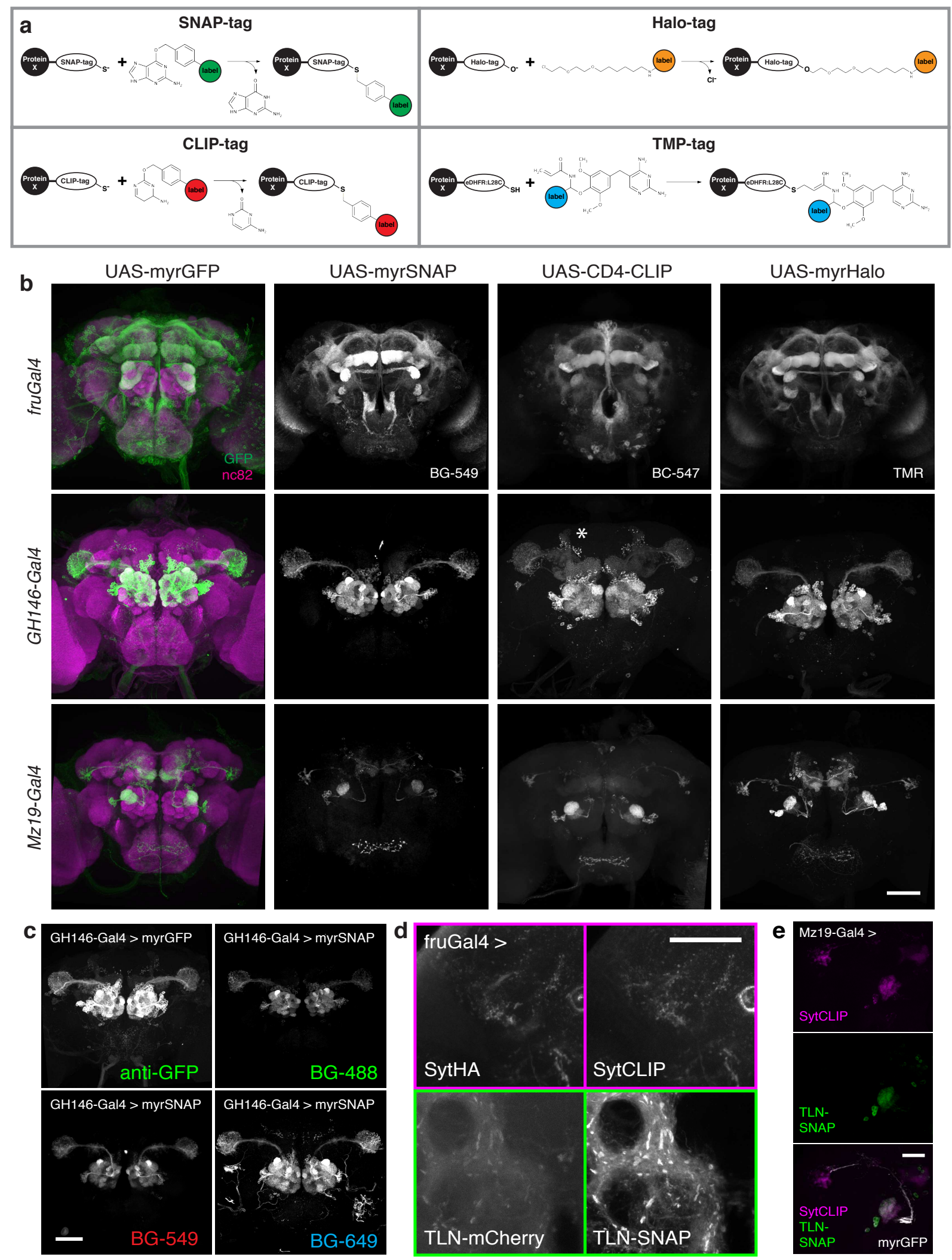

Figure 1: Expression of chemical tags in the fly brain

(a) Chemical labeling chemistries used in this study ${ }^{15,16,19,23}$; see text for description. (b) Expression patterns of membrane-targeted tags in the Drosophila central brain. The panel is arranged in a 3 row $\times 3$ column grid. Rows represent Gal4 driver lines, columns represent reporter constructs. In the first column, nc 82 neuropil counterstaining is shown (magenta). Fluorescent substrates used are indicated for each tag. Note that while myrGFP, myrSNAP and myrHalo are targeted attP insertions, CD4-CLIP is a P element insertion. Due to positional effects, the CD4-CLIP reporter shown here labels the bilateral APL neuron when crossed to GH146-Gal4 (asterisk). (c) Brains of GH146-Gal4 > myrSNAP, myrGFP animals stained with GFP antibody (top, left), BG-488 substrate (top, right), BG549 substrate (bottom, left) or BG-647 substrate (bottom, right). (d) Comparison between established synaptic markers and synaptically targeted chemical tags. Confocal slices from the brains of a fru Gal4 $>$ SytHA, SytCLIP (presynaptic markers, top) and a fru Gal4 $>$ TLN-mCherry (DenMark), TLN-SNAP (somatodendritic markers, bottom) animal are shown. (e) Simultaneous labeling of presynaptic sites and the somatodendritic compartment of Mz19 DA1 projections neurons. Scale bars, $50 \mu \mathrm{m}$. See Table $\mathrm{S} 2$ for abbreviations of substrates. 
bioRxiv preprint doi: https://doi.org/10.1101/005298; this version posted May 19, 2014. The copyright holder for this preprint (which was not certified by peer review) is the author/funder, who has granted bioRxiv a license to display the preprint in perpetuity. It is made available under aCC-BY-ND 4.0 International license.
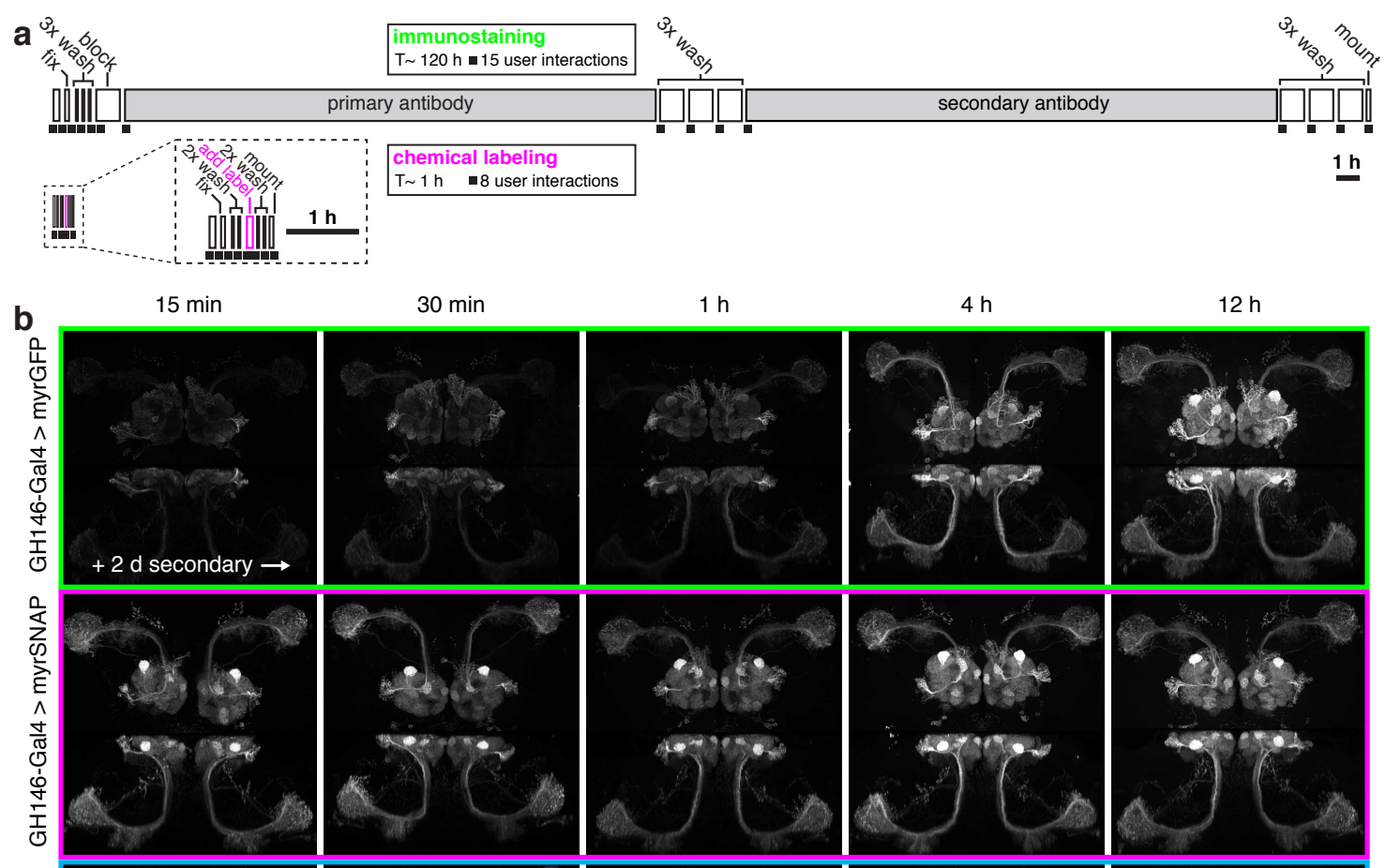

$30 \mathrm{~min}$

$1 \mathrm{~h}$
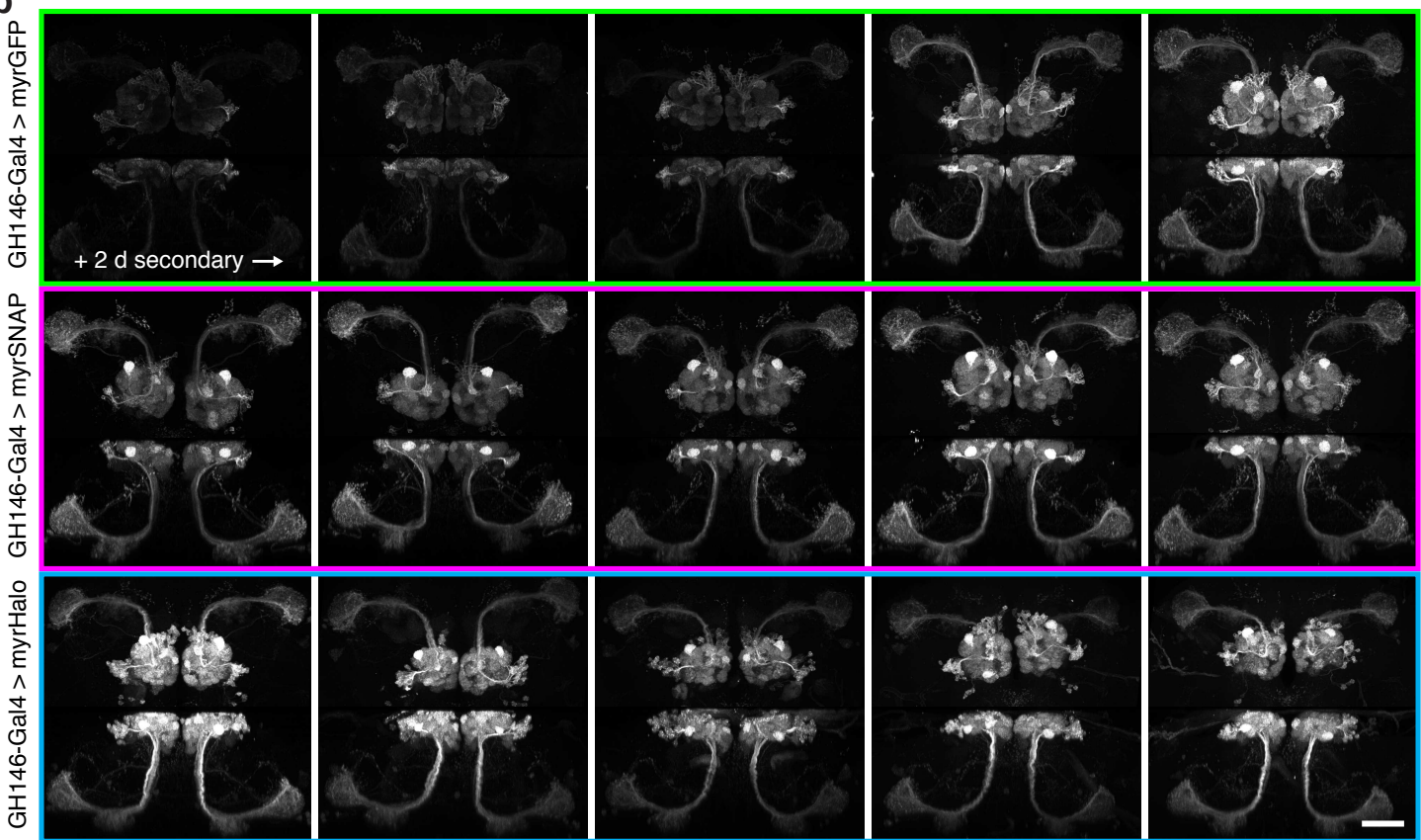

C
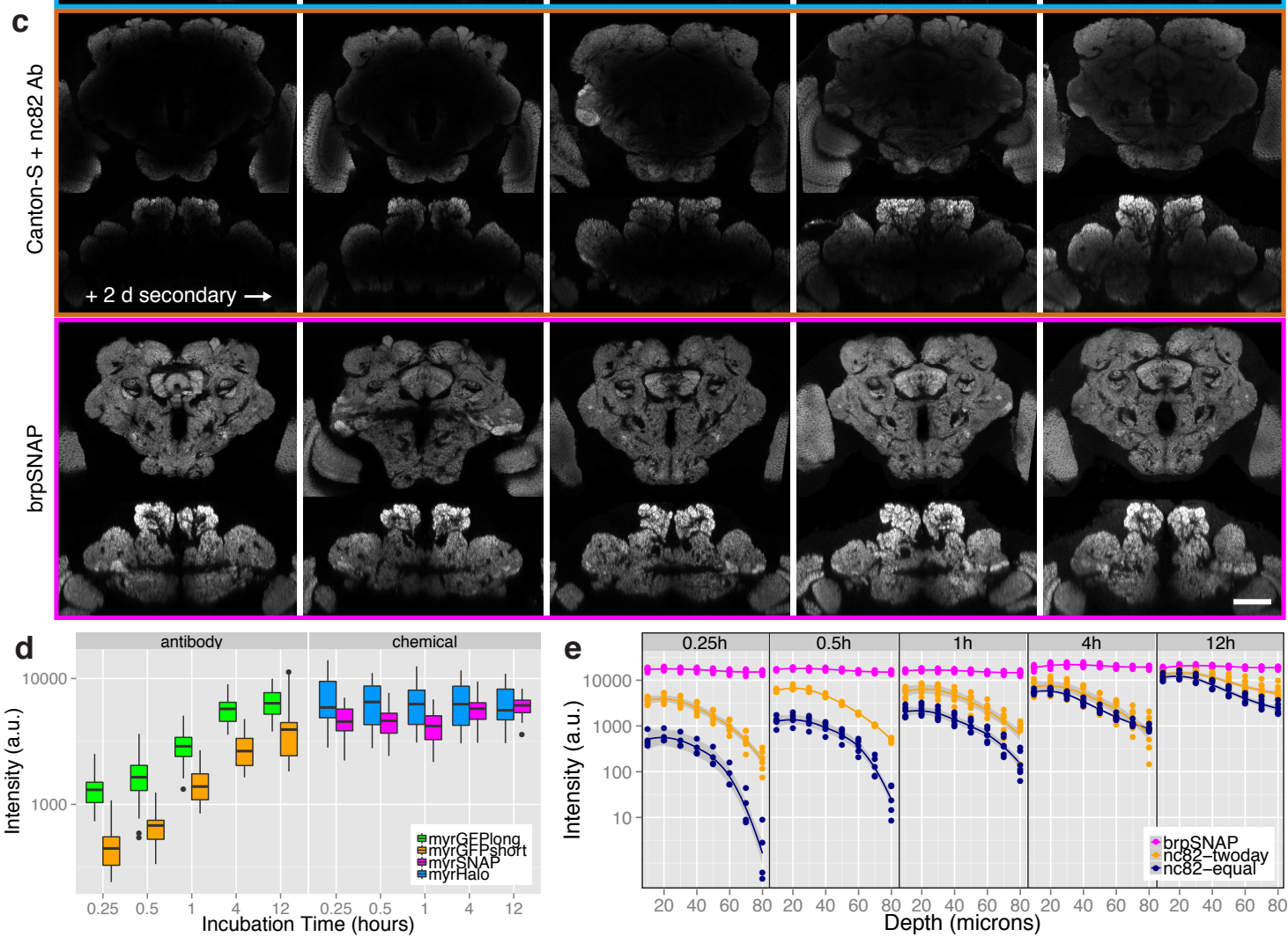

Figure 2: Ultrafast and homogeneous tag-based tissue staining 
bioRxiv preprint doi: https://doi.org/10.1101/005298; this version posted May 19, 2014. The copyright holder for this preprint (which was not certified by peer review) is the author/funder, who has granted bioRxiv a license to display the preprint in perpetuity. It is made available under aCC-BY-ND 4.0 International license.

Figure 2: Ultrafast and homogeneous tag-based tissue staining

(a) Direct comparison of immunostaining and chemical labeling protocols. In these 'ethograms' the length of individual steps is proportional to the time required, and each rectangle represents a manual interaction. Chemical labeling is $>100 \times$ faster $(\sim 1 \mathrm{~h}$ vs. $>100 \mathrm{~h})$ and requires half as many (8 vs. 15) manual handling steps of the sample. (b) Staining time course of GH146 projection neurons using immunostaining against membrane-targeted GFP (top row), chemical labeling using SNAP-tag (middle row) or Halo-tag (bottom row). $z$ - and $y$ - maximum intensity projections from 3D confocal stacks are shown, incubation times are indicated for primary antibody or chemical substrates (GFP antibody, BG-549, Halo-TMR, nc82-antibody). Note that incubation with secondary antibodies was for 2 days. (c) Staining time course of the nc82/Bruchpilot neuropil marker using immunostaining against Brp protein (top row) or chemical labeling using SNAP-tag (bottom row). Single coronal and horizontal confocal slices through the center of the brain are shown. Note that incubation with secondary antibodies was for 2 days. (d) Quantification of signal intensity over time in GH146-Gal4 brains labeled with antibody vs. chemically. Based on brains displayed in (b) and Fig. S5a. Fluorescence was quantified using a GH146 mask (see Fig. S7b and Methods, n = 5-8 brains per condition). (e) Quantification of labeling intensity (see Methods) at different depths from the brain surface in brains labeled with nc 82 antibody vs. chemically (using brp-SNAP). Secondary antibodies for nc 82 immunostainings were either incubated for $2 \mathrm{~d}$ ('nc82-twoday') or for the same duration as primary antibodies ('nc82-equal'). Five different time points are shown. Scale bars, $50 \mu \mathrm{m}$. 
bioRxiv preprint doi: https://doi.org/10.1101/005298; this version posted May 19, 2014. The copyright holder for this preprint (which was not certified by peer review) is the author/funder, who has granted bioRxiv a license to display the preprint in perpetuity. It is made available under aCC-BY-ND 4.0 International license.

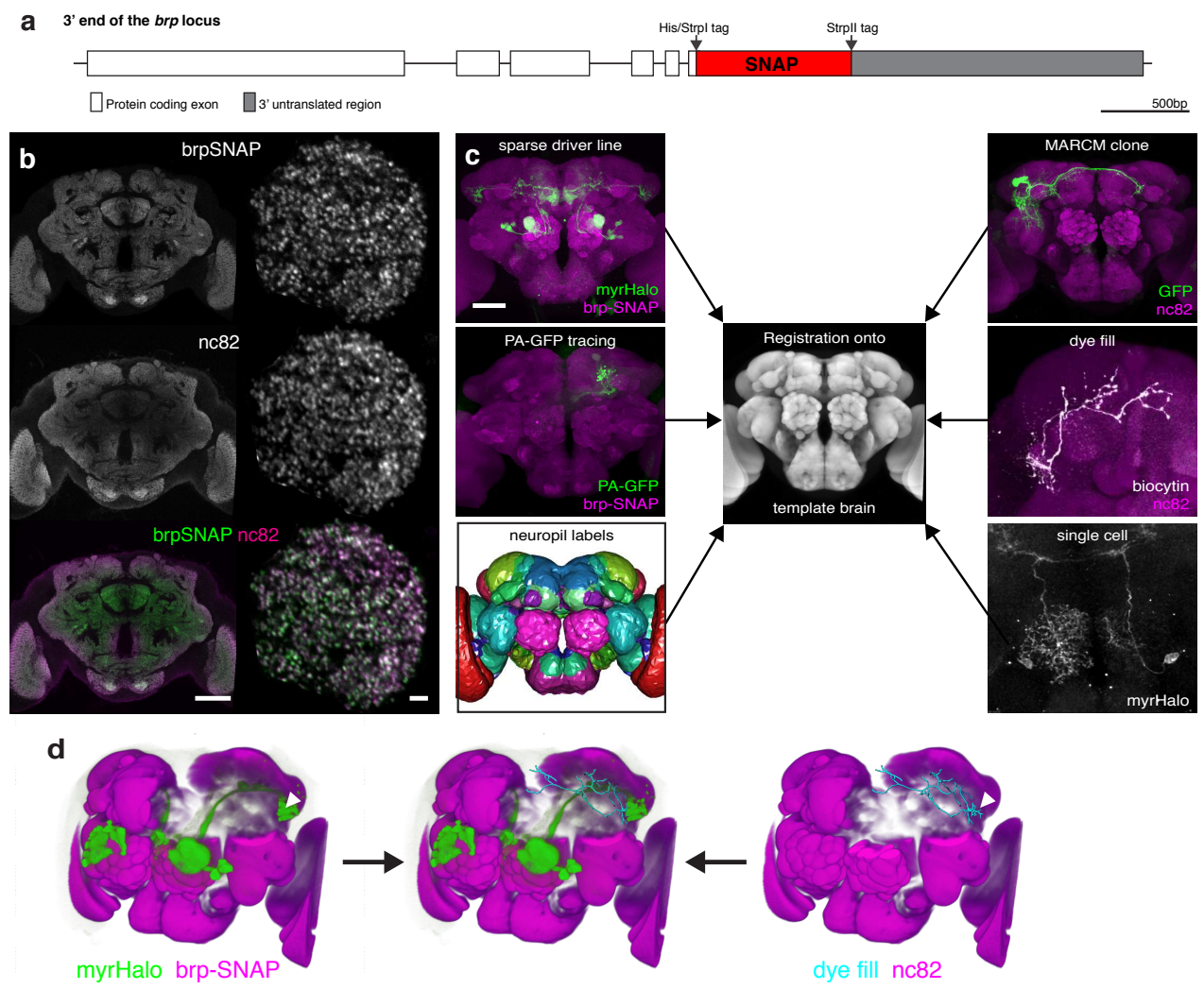

Figure 3: Key applications for chemical labeling in Drosophila neurobiology

(a) Map of the brp-SNAP knock-in. (b) nc82 and brp-SNAP signals co-localize in a brp-SNAP/+ brain simultaneously labeled with BG-549 and nc82 antibody. Single coronal slices through the middle of the brain (left) and through a deconvolved image stack of the DA1 glomerulus (right) are shown. Note the more even brp-SNAP staining in the center of the brain. Scale bars, $50 \mu \mathrm{m}$ (top), $2 \mu \mathrm{m}$ (bottom). (c) Chemically labeled neurons can be successfully registered onto a template using the brp-SNAP neuropil counterstaining. This allows direct comparison with neurons from other sources, such as stochastic labeling (MARCM), PA-GFP tracing experiments, whole-cell recordings or sparse driver lines (the Mz19-Gal4 expression pattern and a single neuron from GMR14C11-Gal4 are shown). Scale bar, $50 \mu \mathrm{m}$. (d) Overlay of DA1 projection neurons (green) from a chemically labeled brp-SNAP, Mz19-Gal4 > myrHalo brain (left) overlaid with a dye-filled third-order olfactory neuron (cyan) from an nc82-stained brain (right) after registration. Notice the overlap between axon terminals and dendritic arbor (white arrows). 
bioRxiv preprint doi: https://doi.org/10.1101/005298; this version posted May 19, 2014. The copyright holder for this preprint (which was not certified by peer review) is the author/funder, who has granted bioRxiv a license to display the preprint in perpetuity. It is made available under aCC-BY-ND 4.0 International license.

Table 1: Comparison of immunostainings with chemical labeling

\begin{tabular}{|c|c|c|}
\hline & immunostaining & chemical labeling \\
\hline duration & hours-days & minutes-hours \\
\hline user interactions & $>15^{(\mathrm{a})}$ & 8 \\
\hline substrate size & $\sim 150 \mathrm{kDa}$ & $\sim 1 \mathrm{kDa}$ \\
\hline staining steps & $2^{(\mathrm{b})}$ & 1 \\
\hline blocking & Yes & No \\
\hline background & often high & low \\
\hline epitope stability & variable & high \\
\hline cell permeable & No & Yes/No \\
\hline No. of channels & many & 4 (currently) \\
\hline transgenics & No & Yes \\
\hline stoichiometry $^{(c)}$ & $>1: 1$ & $1: 1$ \\
\hline
\end{tabular}

Comparing antibody-based and tag-based staining approaches. Staining durations indicated are typical for thick tissue samples (hundreds of $\mu \mathrm{m}$ to several mm). Notes: (a) Using standard immunostaining protocol for Drosophila brains (see text for references). (b) Fluorophore-coupled primary antibodies are sometimes used, reducing the number of staining steps to 1. (c) i.e. label-to-tag ratio. 
bioRxiv preprint doi: https://doi.org/10.1101/005298; this version posted May 19, 2014. The copyright holder for this preprint (which was not certified by peer review) is the author/funder, who has granted bioRxiv a license to display the preprint in perpetuity. It is made available under aCC-BY-ND 4.0 International license.

\section{SUPPLEMENT}


bioRxiv preprint doi: https://doi.org/10.1101/005298; this version posted May 19,2014. The copyright holder for this preprint (which was not certified by peer review) is the author/funder, who has granted bioRxiv a license to display the preprint in perpetuity. It is made available under aCC-BY-ND 4.0 International license.

Table S1: Transgenic fly lines generated in this study

\begin{tabular}{|c|c|c|c|c|}
\hline line & tag & available insertions & description & tag location \\
\hline \hline UAS-CD4-CLIP & CLIPf & 2,3 & plasma membrane targeting (myristoylation signal) \\
\hline UAS-myr-SNAP & SNAPf & 2 (attP40), 3 (attP2) & plasma membrane targeting (myristoylation signal) \\
\hline UAS-myr-TMP & TMP & 2 (attP40), 3 (attP2) & plasma membrane targeting (myristoylation signal) \\
\hline UAS-myr-Halo & Halo & 2 (attP40), 3 (attP2) & intra & intra \\
\hline UAS-Syt-SNAP & SNAPm & 2,3 & presynaptic label, fusion to Drosophila synaptotagmin (see ref. [ ${ }^{29}$ ]) & intra \\
\hline UAS-Syt-CLIP & CLIPm & 2,3 & presynaptic label, fusion to Drosophila synaptotagmin (see ref. [ ${ }^{29}$ ]) & intra \\
\hline UAS-SNAP-syb & SNAPf & 2,3 & presynaptic label, fusion to Drosophila n-synaptobrevin (see ref. [ ${ }^{29}$ ]) & intra \\
\hline UAS-CLIP-syb & CLIPf & 2,3 & presynaptic label, fusion to Drosophila n-synaptobrevin (see ref. [ ${ }^{29}$ ]) & intra \\
\hline UAS-TLN-SNAP & SNAPm & 2,3 & postsynaptic label, fusion to mouse ICAM-5 (see ref. [ ${ }^{31}$ ]) & intra \\
\hline UAS-TLN-CLIP & CLIPm & 2,3 & postsynaptic label, fusion to mouse ICAM-5 (see ref. [ ${ }^{31}$ ]) & intra \\
\hline brp-SNAP & SNAPf & 2 & targeted (MiMIC-based) insertion into $b r p$ locus & intra \\
\hline brp-SNAP; myrHalo & SNAPf; Halo & $2 ; 3$ (attP2) & targeted (MiMIC-based) insertion into brp locus; membrane label & intra \\
\hline
\end{tabular}

Table S1: Transgenic fly lines generated in this study

Transgenic fly reporter lines generated in this study are listed. P element insertions were mapped to chromosomes 2 or 3, respectively; insertions into attP sites are indicated in parentheses. 
bioRxiv preprint doi: https://doi.org/10.1101/005298; this version posted May 19, 2014. The copyright holder for this preprint (which was not certified by peer review) is the author/funder, who has granted bioRxiv a license to display the preprint in perpetuity. It is made available under aCC-BY-ND 4.0 International license.

Table S2: Commercial and custom fluorophore-coupled substrates

Commercially available, fluorophore-coupled substrates for SNAP-, CLIP-, Halo- and TMP-tag are listed. 
bioRxiv preprint doi: https://doi org/10.1101/005298; this version posted May 19,2014. The copyright holder for this preprint (which was not

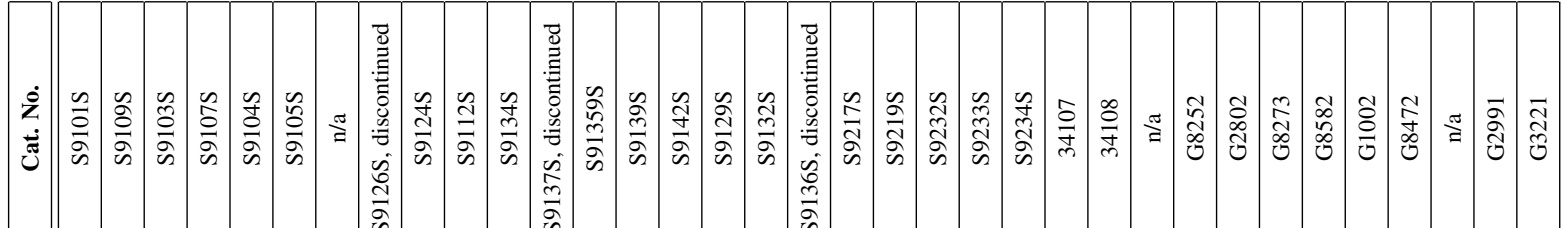

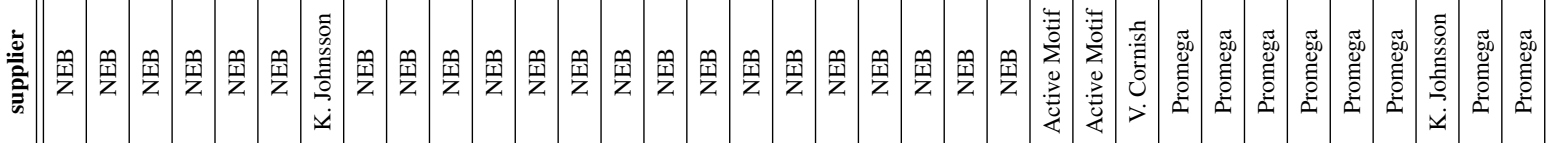

苋

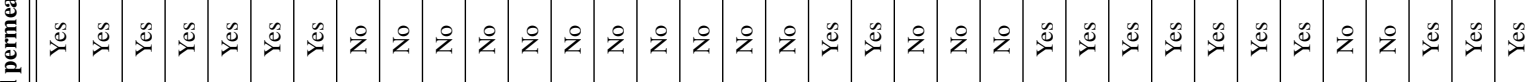
这

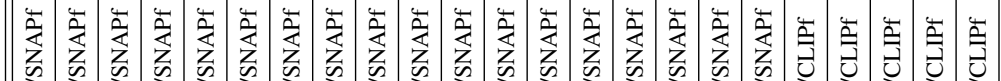

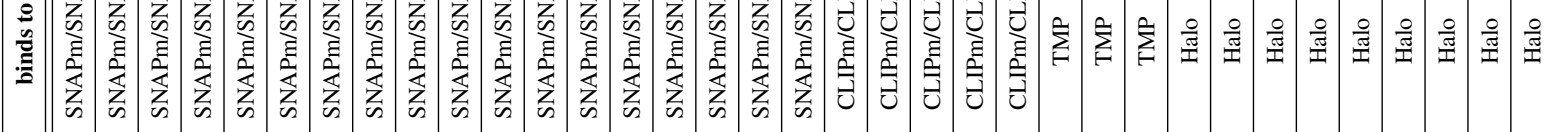

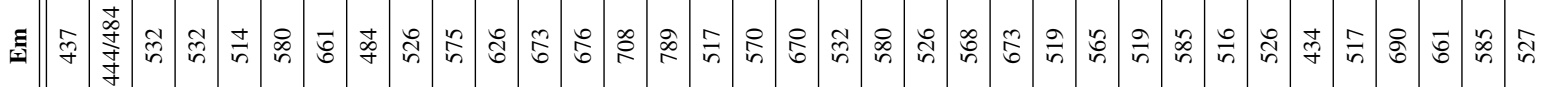

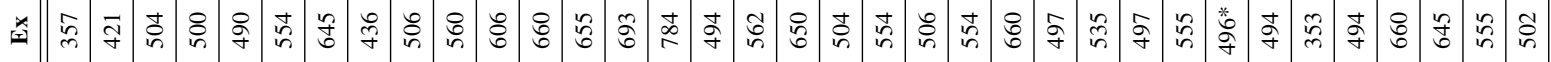

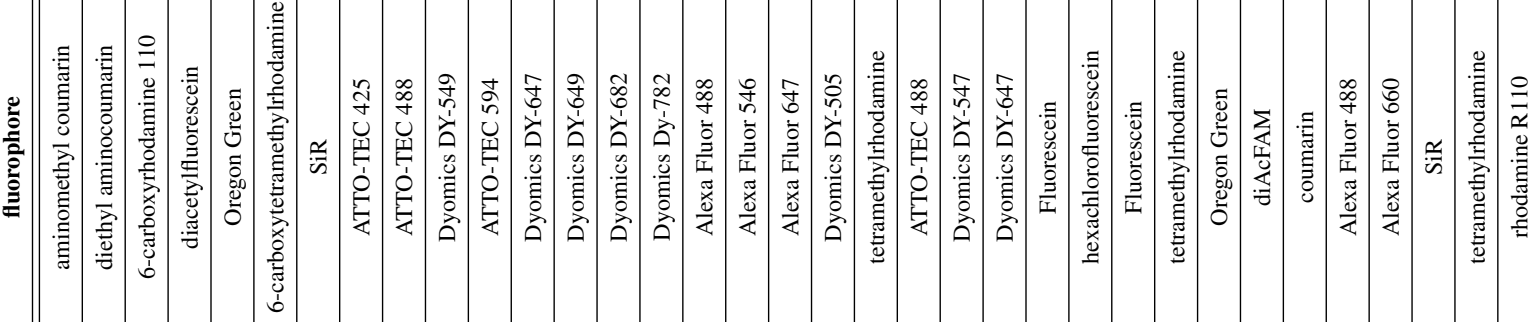

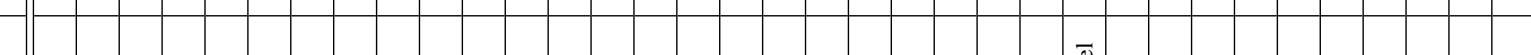

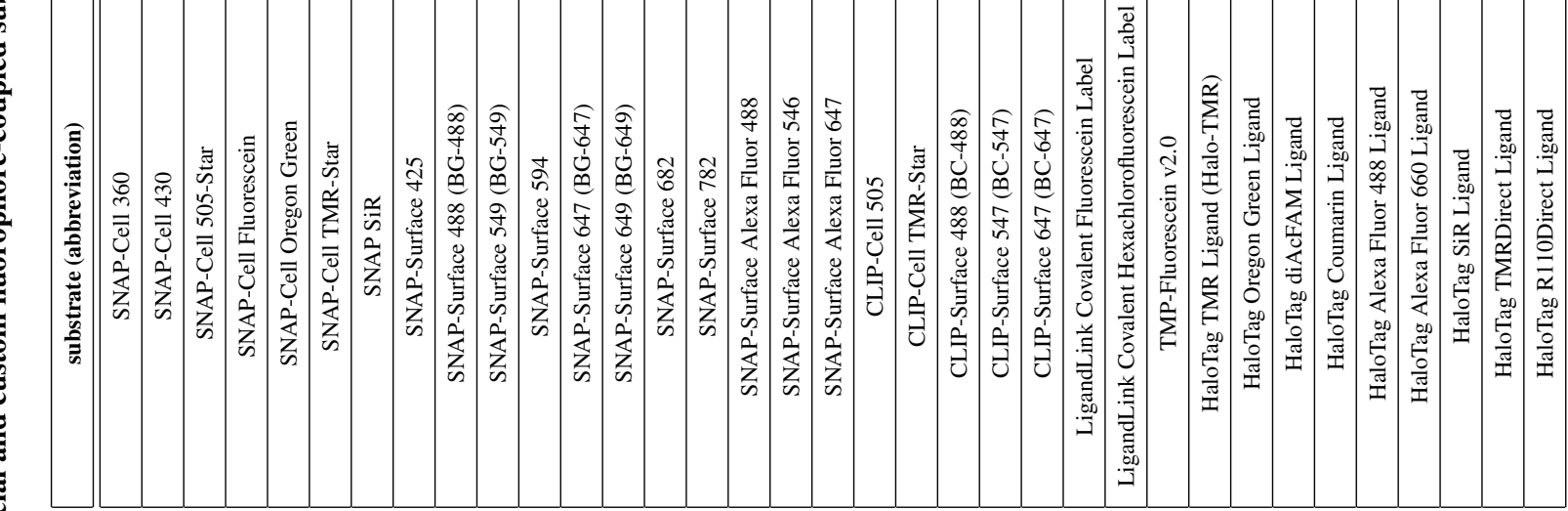


bioRxiv preprint doi: https://doi.org/10.1101/005298; this version posted May 19, 2014. The copyright holder for this preprint (which was not certified by peer review) is the author/funder, who has granted bioRxiv a license to display the preprint in perpetuity. It is made available under aCC-BY-ND 4.0 International license.

Figure S1: Additional chemical tag reporter fly lines
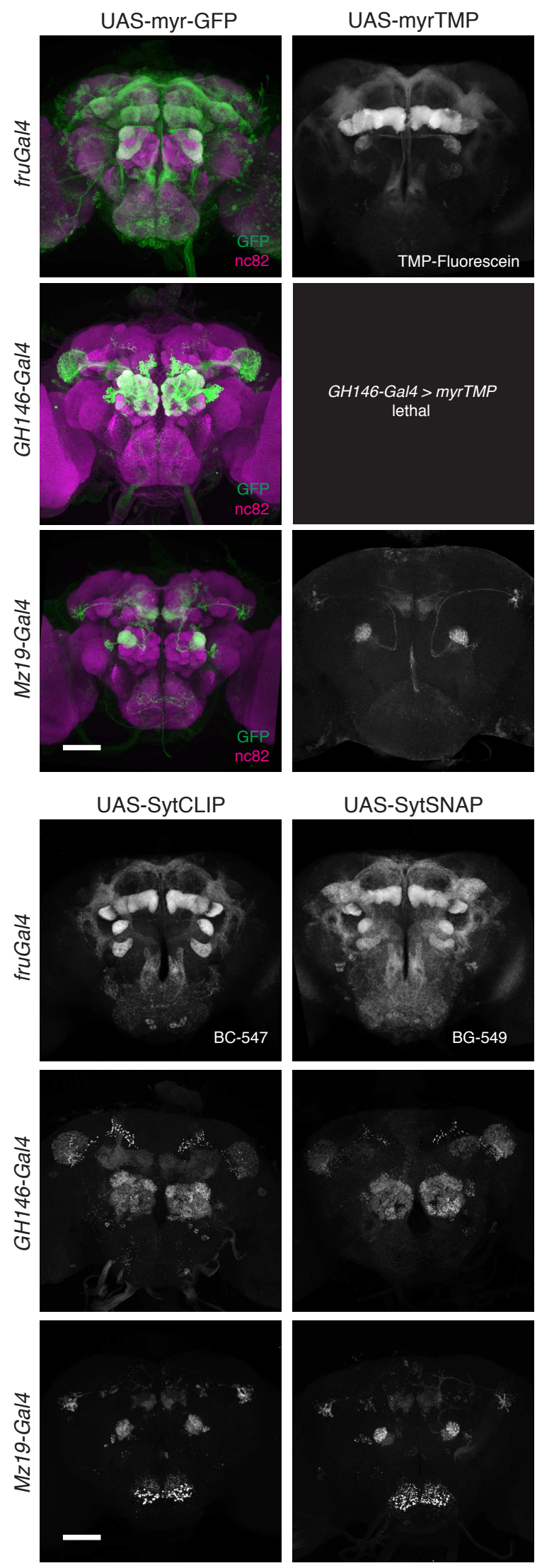

UAS-SytSNAP
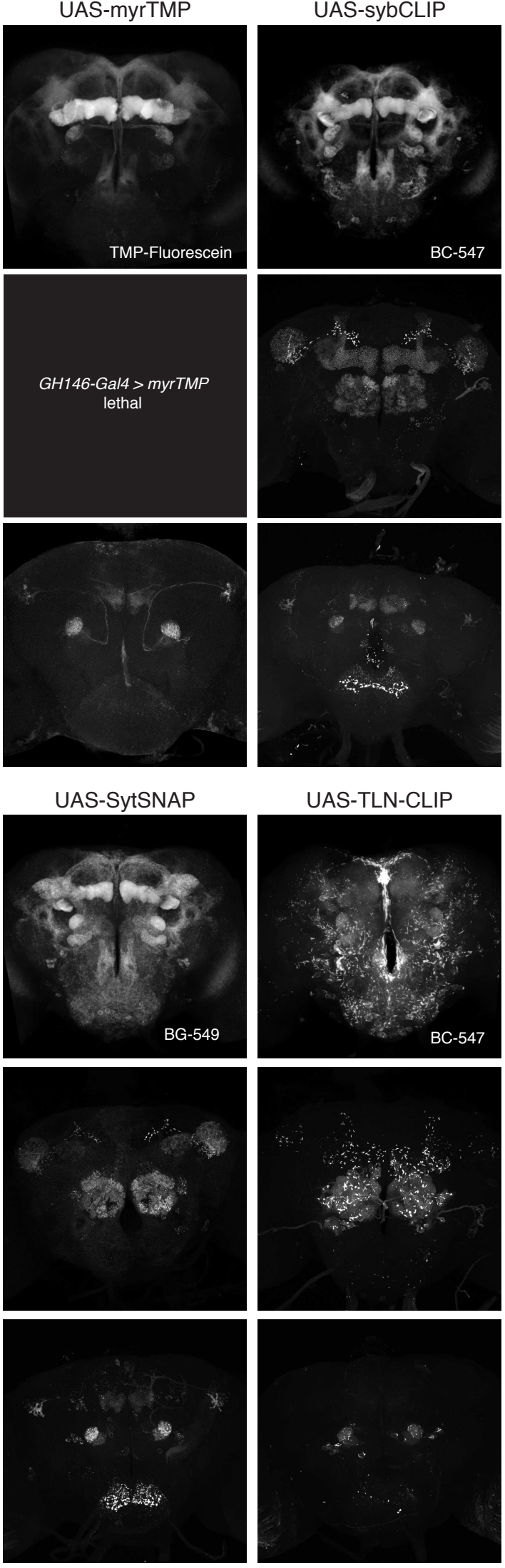

UAS-TLN-CLIP
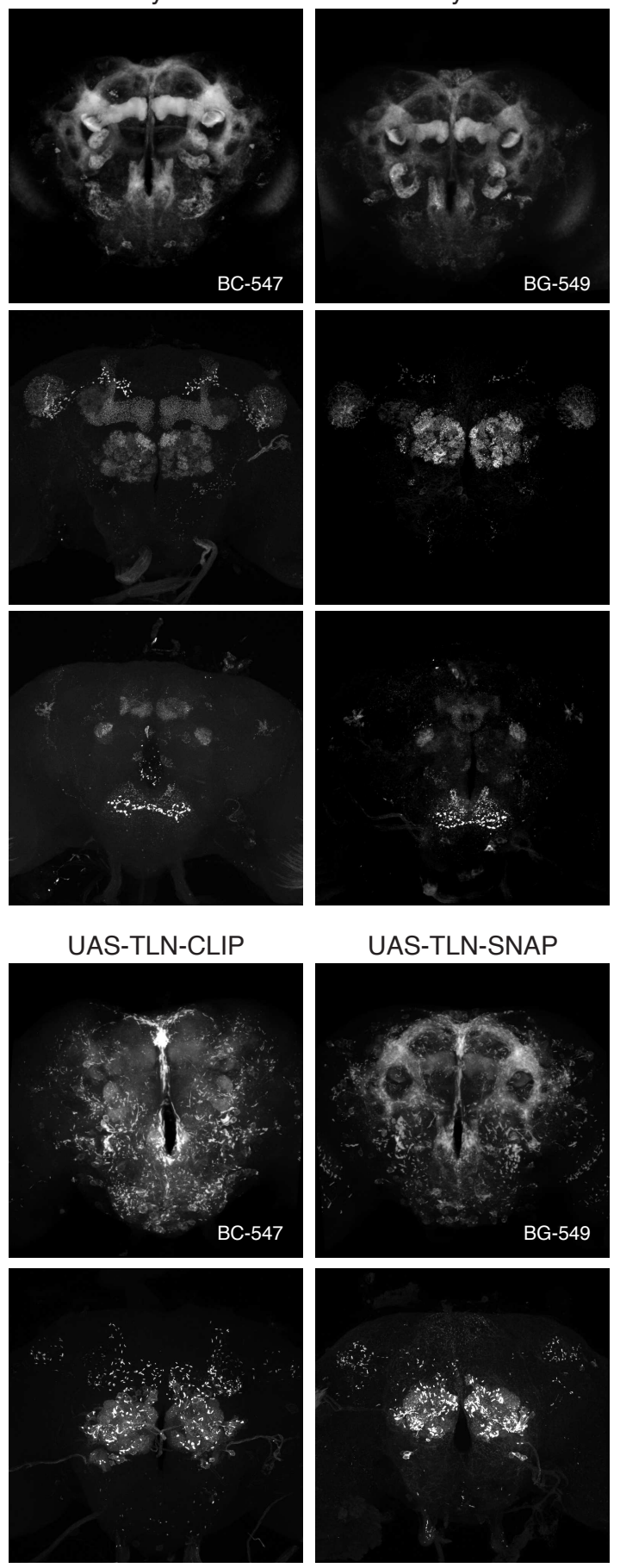

UAS-TLN-SNAP
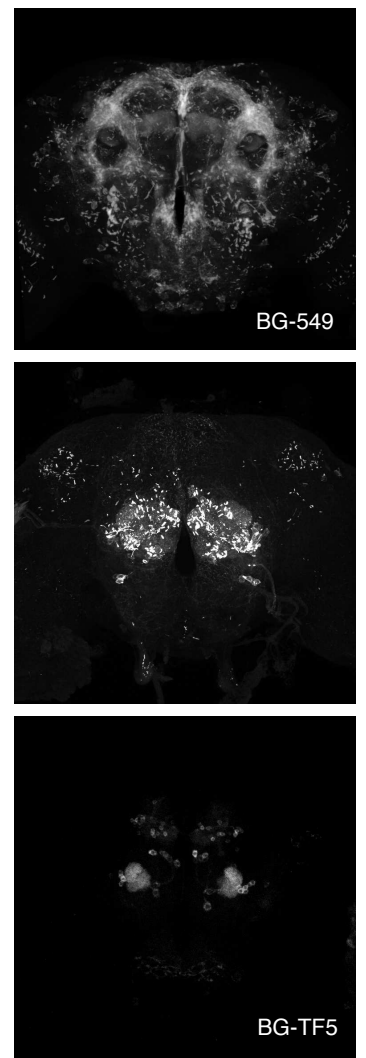

Expression patterns of additional selected chemical tagging constructs in flies. As in Fig. 1, three different driver lines were used. Images are standard deviation projections from 3D confocal stacks, used substrates are indicated in each image. Scale bars, $50 \mu \mathrm{m}$. 
bioRxiv preprint doi: https://doi.org/10.1101/005298; this version posted May 19, 2014. The copyright holder for this preprint (which was not certified by peer review) is the author/funder, who has granted bioRxiv a license to display the preprint in perpetuity. It is made available under aCC-BY-ND 4.0 International license.

Figure S2: Cross-reactivity of tag-substrate combinations used in this study

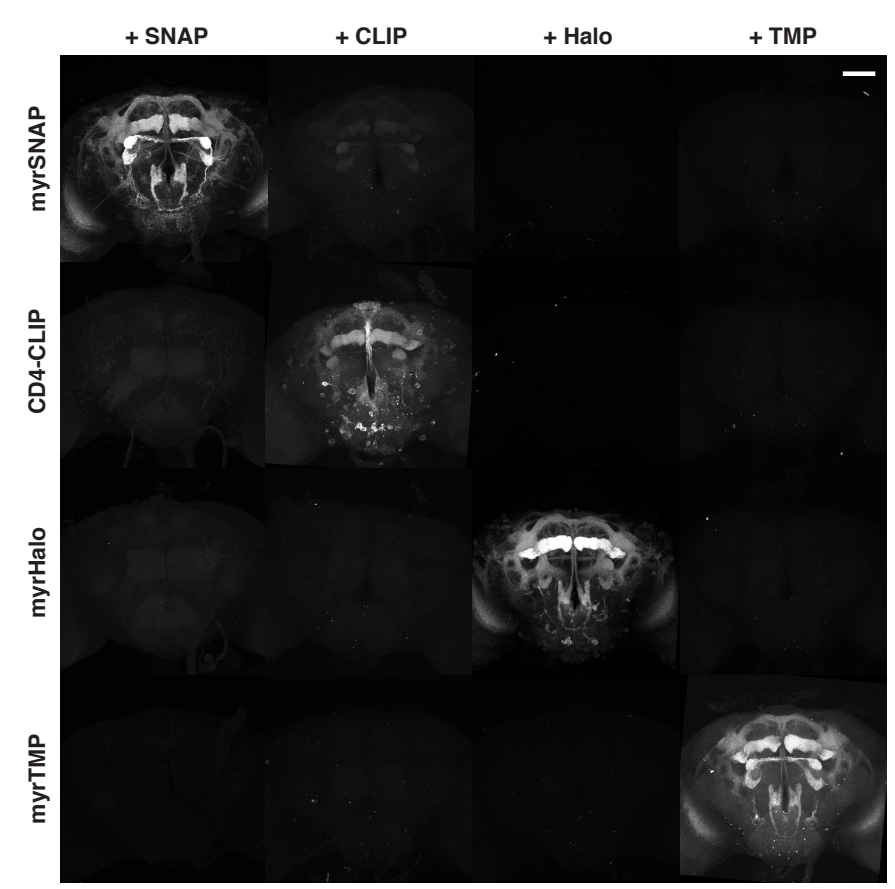

Tag-substrate cross-reactivity matrix. Brains in which fru Gal4 drives expression of membrane-bound tags were incubated with matching and non-matching substrates (SNAP, BG-549 $1 \mu \mathrm{M}$, CLIP, BC-488 $1 \mu \mathrm{M}$, Halo, Halo-TMR $500 \mathrm{nM}$, TMP, TMP-Fluorescein 500 nM). No cross-reactivity can be detected, with the exception of CLIP substrate with SNAP-tag. Scale bar, $50 \mu \mathrm{m}$. 
bioRxiv preprint doi: https://doi.org/10.1101/005298; this version posted May 19, 2014. The copyright holder for this preprint (which was not certified by peer review) is the author/funder, who has granted bioRxiv a license to display the preprint in perpetuity. It is made available under aCC-BY-ND 4.0 International license.

Figure S3: Fixation-resistance of enzyme tags

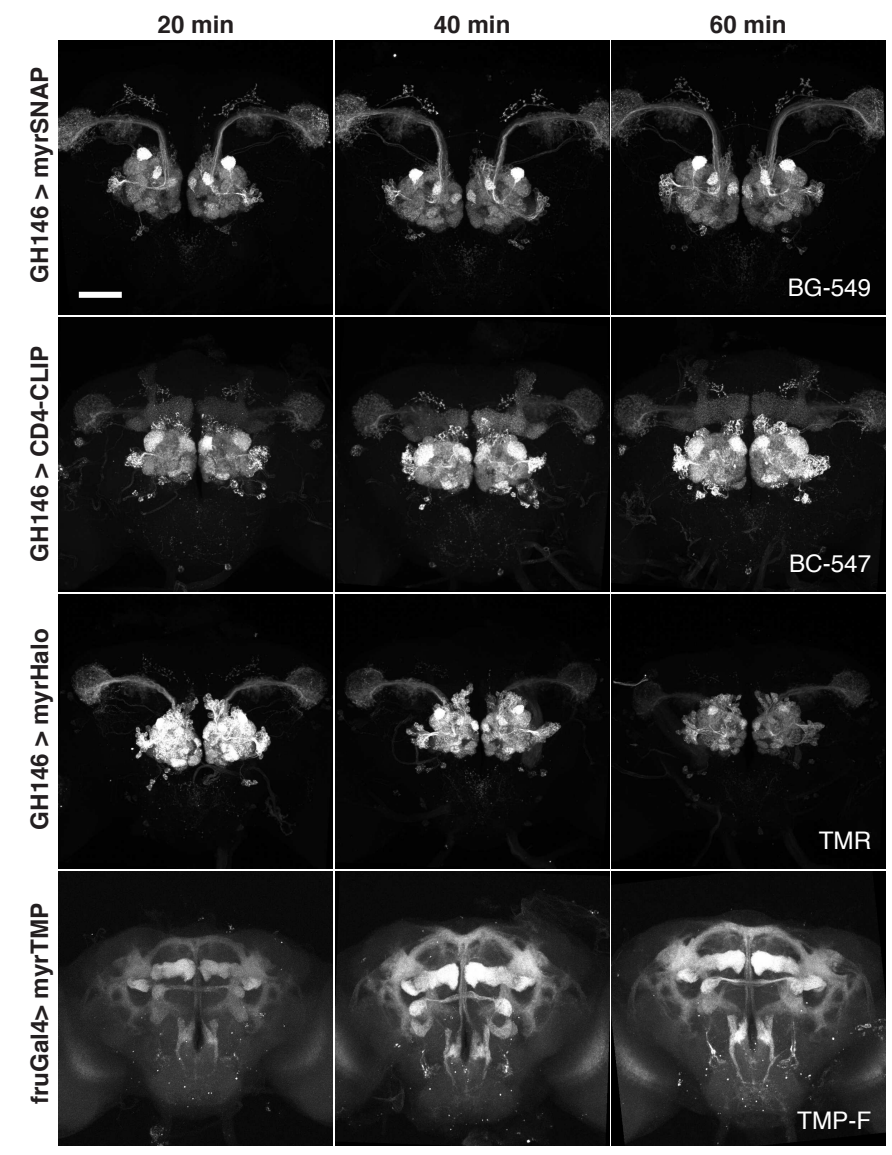

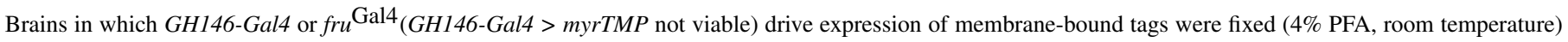
for the indicated time, permeabilized and stained (all substrates $1 \mu \mathrm{M}, 30$ min incubation). Note that while SNAP- and CLIP-tag labeling intensities remain largely constant wtih increasing fixation times, Halo-tag labeling intensity decreases and TMP-tag labeling intensity increases. Scale bar, $50 \mu \mathrm{m}$. 
bioRxiv preprint doi: https://doi.org/10.1101/005298; this version posted May 19, 2014. The copyright holder for this preprint (which was not certified by peer review) is the author/funder, who has granted bioRxiv a license to display the preprint in perpetuity. It is made available under aCC-BY-ND 4.0 International license.

Figure S4: Generation of brp-SNAP

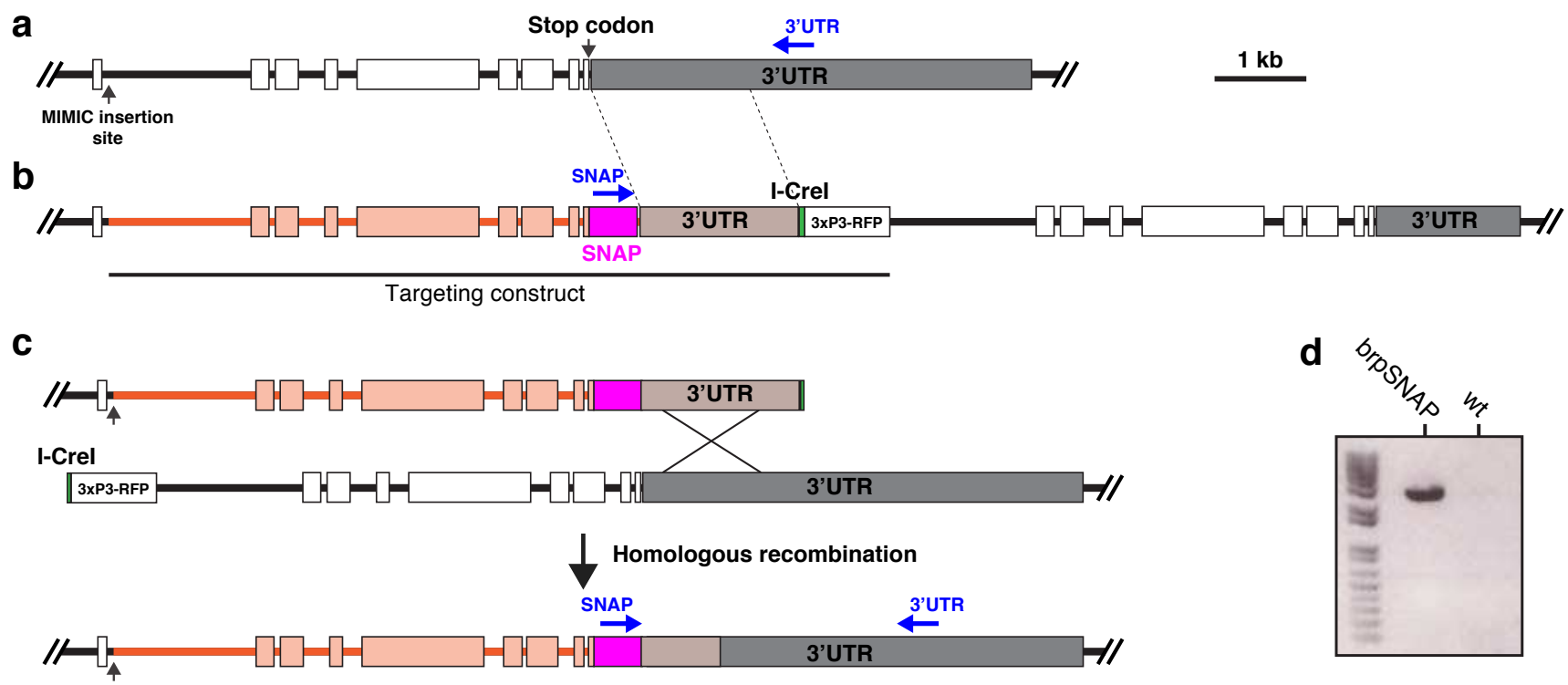

Generation of the brp-SNAP knock-in allele. (a) Schematic showing the organization of the 3' end of the brp locus and the location of the MiMIC insertion. (b) Schematic showing the integrated targeting construct and the resulting duplication. (c) Resolution of the duplication. The double strand breakage produced by I-CreI is repaired by homologous recombination, resulting in the loss of the RFP marker and the reduction of the duplication. (d) PCR with primers 'SNAP' and '3'UTR' produces a band in recombinant (brpSNAP), but not wild-type (wt) flies. 
bioRxiv preprint doi: https://doi.org/10.1101/005298; this version posted May 19, 2014. The copyright holder for this preprint (which was not certified by peer review) is the author/funder, who has granted bioRxiv a license to display the preprint in perpetuity. It is made available under aCC-BY-ND 4.0 International license.

Figure S5: Comparison of neuropil antibody staining and labeling using the brp-SNAP transgene

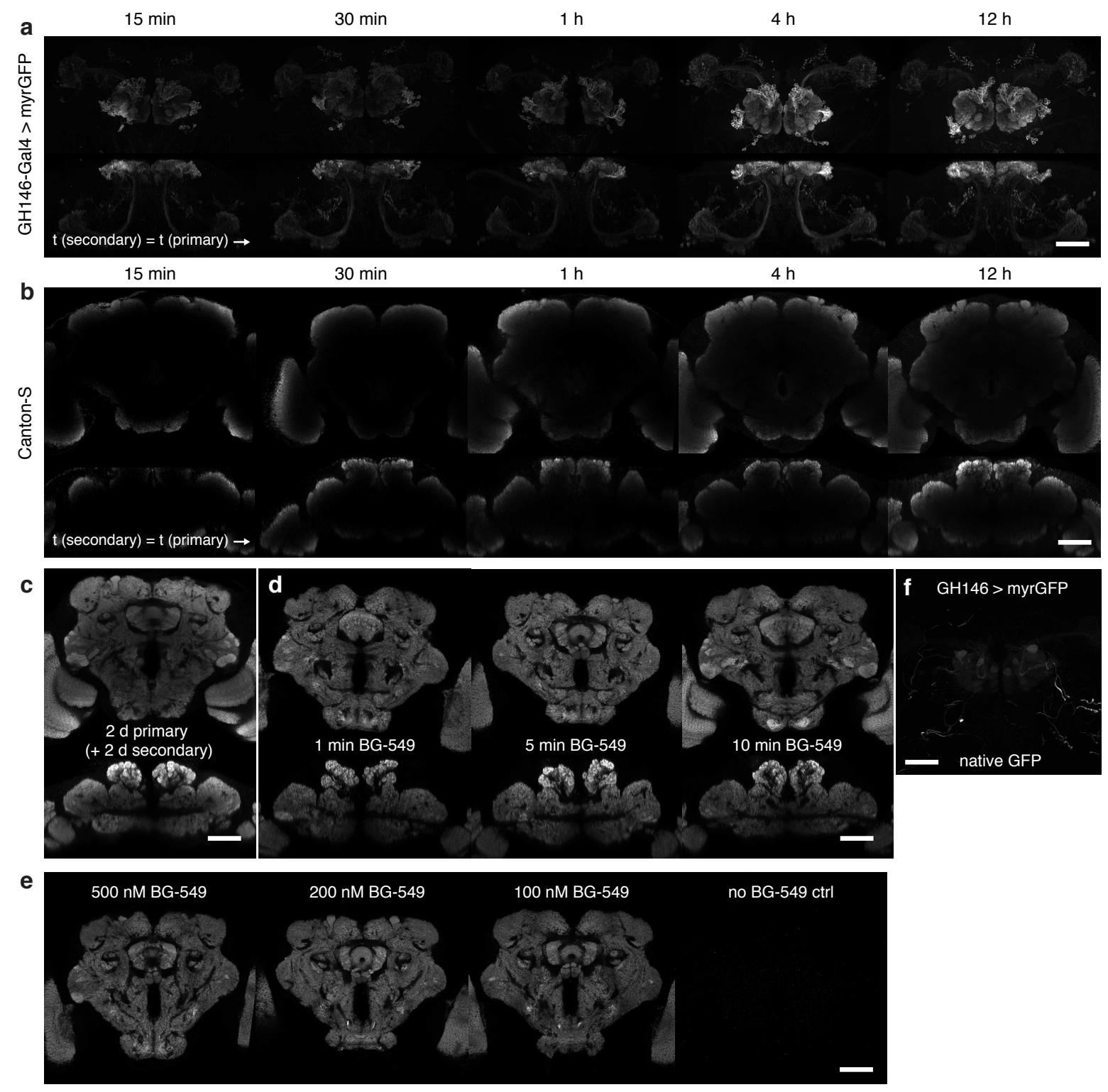

Figure S5: Ultrafast and homogeneous tag-based tissue staining (continued)

(a-b) Staining time course of GH146-Gal4 > myrGFP brains with anti-GFP antibody (a) and staining time course of the nc82/Bruchpilot neuropil marker using immunostaining against Brp protein (b). ( $z$ - and $x$-) Maximum projections from 3D confocal stacks are shown in (a), while single coronal (top) and horizontal (bottom) confocal slices through the center of the brain are shown in (b), incubation times are indicated. Note that in contrast to the stainings shown in Fig. 2 (secondary antibodies incubated for $2 \mathrm{~d}$ in each case), incubation times are identical for primary and secondary antibodies in (a) and (b). (c) Example of nc82stained brain after full ( $2 \mathrm{~d}$ primary antibody $+2 \mathrm{~d}$ secondary antibody) immunostaining protocol. A coronal (top) and a horizontal slice (bottom) from a 3D confocal stack are shown. (d) brp-SNAP brains after short incubations with BG-549. Coronal (top) and horizontal (bottom) slices through the middle of the brain are shown. Note that homogeneous staining is visible already after 1 min. (e) brp-SNAP brain stained with lower concentrations of BG-549 substrate and negative control without substrate. Incubation time was 15 min. Coronal slices through the middle of the brain are shown. (f) Unstained GH146-Gal4 > myrGFP brain imaged after fixation. Most endogenous GFP fluorescence is quenched. Scale bars, $50 \mu \mathrm{m}$. 
bioRxiv preprint doi: https://doi.org/10.1101/005298; this version posted May 19, 2014. The copyright holder for this preprint (which was not certified by peer review) is the author/funder, who has granted bioRxiv a license to display the preprint in perpetuity. It is made available under aCC-BY-ND 4.0 International license.

Figure S6: Sequential labeling reveals binding kinetics
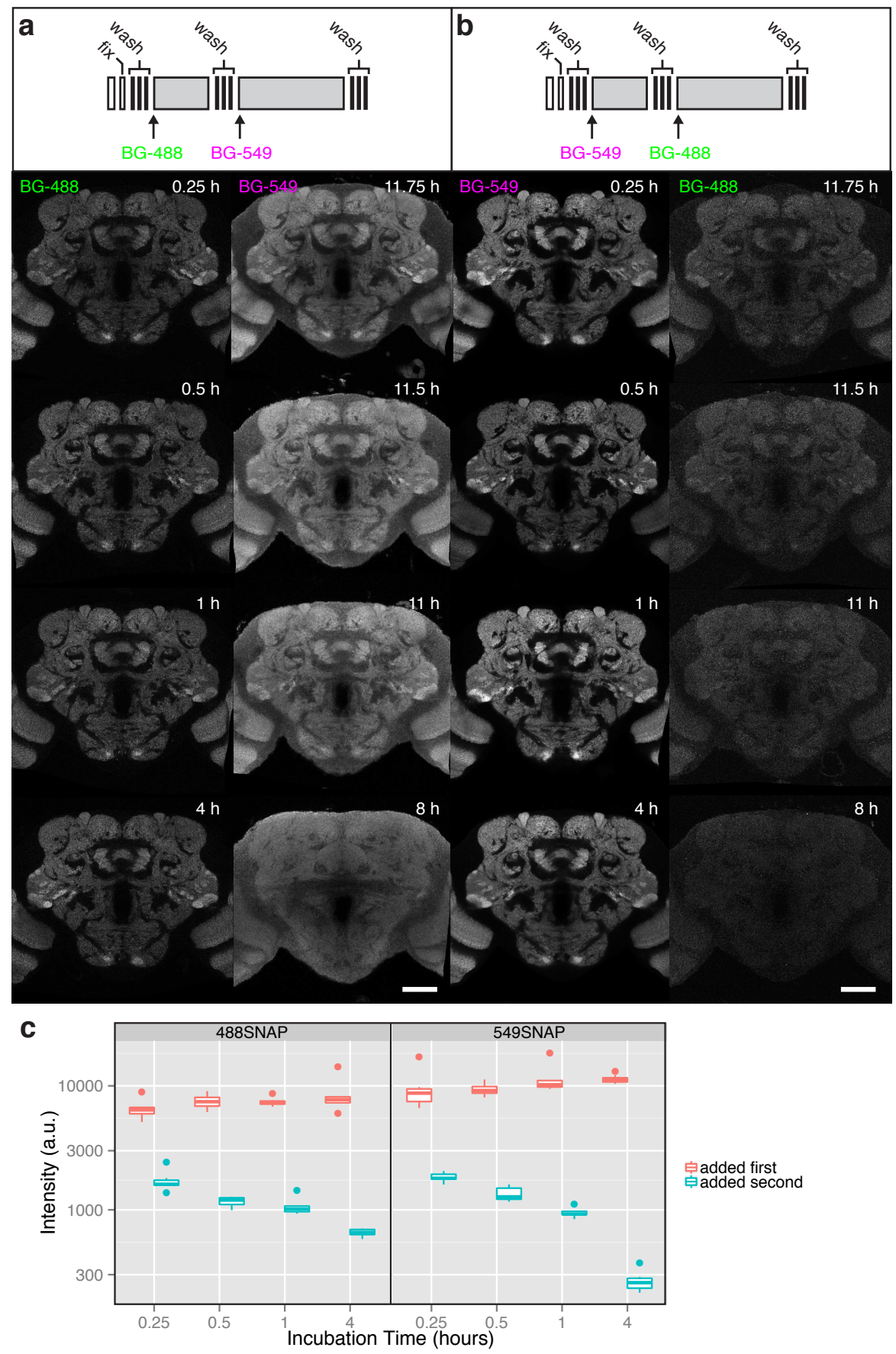

Sequential incubation of brp-SNAP brains with BG-488 followed by BG-549 (a) or BG-549 followed by BG-488 (b). The first substrate was added for the indicated time $(0.25 \mathrm{~h}, 0.5 \mathrm{~h}, 1 \mathrm{~h}, 4 \mathrm{~h})$, after a washing step the second substrate was added for a total staining time of $12 \mathrm{~h}$. (c) Quantification of labeling achieved with BG-488 (left panel) or BG-549 (right panel) using a neuropil mask (see Fig. S7 and methods). Scale bars, $50 \mu \mathrm{m}$. 
bioRxiv preprint doi: https://doi.org/10.1101/005298; this version posted May 19,2014. The copyright holder for this preprint (which was not certified by peer review) is the author/funder, who has granted bioRxiv a license to display the preprint in perpetuity. It is made available under aCC-BY-ND 4.0 International license.

Figure S7: Masks used for quantification of labeling intensity in fly brains

a whole brain neuropil

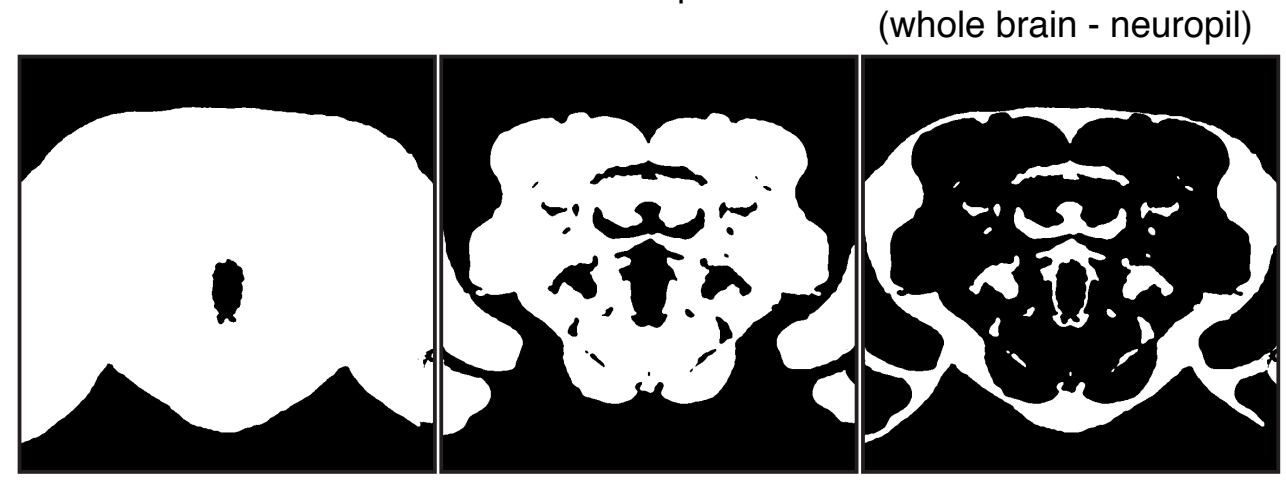

b

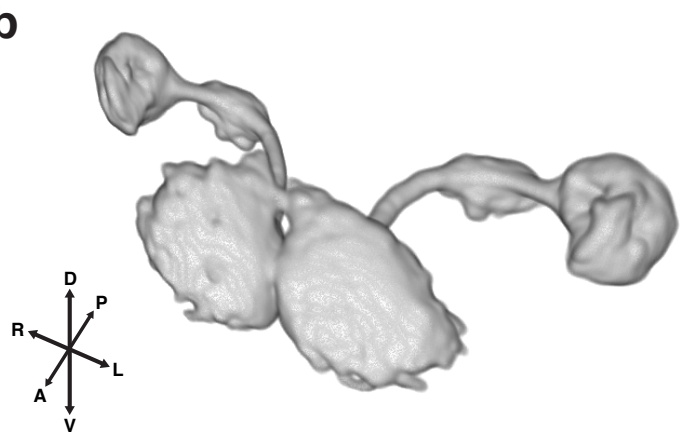

(a) Masks used for quantification of labeling in brains stained against nc82- or brp-SNAP. Single coronal slices through the 3D masks are shown. (b) Volumerendering of mask used for quantification of labeling in GH146-Gal4 brains. 